JOURNAL OF THE

AMERICAN MATHEMATICAL SOCIETY

Volume 16, Number 3, Pages 461-494

S 0894-0347(03)00422-3

Article electronically published on January 24, 2003

\title{
MULTIDIMENSIONAL TRANSONIC SHOCKS AND FREE BOUNDARY PROBLEMS FOR NONLINEAR EQUATIONS OF MIXED TYPE
}

\author{
GUI-QIANG CHEN AND MIKHAIL FELDMAN
}

\section{INTRODUCTION}

We are concerned with the existence and stability of multidimensional transonic shocks for the Euler equations for steady potential compressible fluids. The Euler equations, consisting of the conservation law of mass and the Bernoulli law for the velocity, can be written as the following second-order nonlinear equation of mixed elliptic-hyperbolic type for the velocity potential $\varphi: \Omega \subset \mathbf{R}^{n} \rightarrow \mathbf{R}$ :

$$
\operatorname{div}\left(\rho\left(|D \varphi|^{2}\right) D \varphi\right)=0,
$$

where the density function $\rho\left(q^{2}\right)$ is

$$
\rho\left(q^{2}\right)=\left(1-\theta q^{2}\right)^{\frac{1}{2 \theta}}
$$

with $\theta=\frac{\gamma-1}{2}>0$ for the adiabatic exponent $\gamma>1$.

The second-order nonlinear equation (1.1) is strictly elliptic at $D \varphi$ with $|D \varphi|=q$ if

$$
\rho\left(q^{2}\right)+2 q^{2} \rho^{\prime}\left(q^{2}\right)>0
$$

and is strictly hyperbolic if

$$
\rho\left(q^{2}\right)+2 q^{2} \rho^{\prime}\left(q^{2}\right)<0 .
$$

The elliptic regions of equation (1.1) correspond to the subsonic flow, and the hyperbolic regions of (1.1) correspond to the supersonic flow.

Some efforts have been made in solving the nonlinear equation (1.1) of mixed type. Shiffman [30], Bers [5], and Finn-Gilbarg [16] proved the existence and uniqueness of solutions for the problem of subsonic flows of (1.1) passing an obstacle and showed that, if the uniform outflow speed at infinity is sufficiently subsonic, then there exists a unique subsonic solution of this problem, in which the nonlinear equation (1.1) is uniformly elliptic; also see Dong [15] for further results. When the uniform outflow speed at infinity is near sonic, Morawetz in [27. showed that the flows of (1.1) past the obstacle may contain transonic shocks in general. One exception is that, when the obstacle forms a wedge or conical body with a sharp

Received by the editors October 17, 2001.

2000 Mathematics Subject Classification. Primary 35M10, 35J65, 35R35, 76H05, 76L05, 35B 45.

Key words and phrases. Transonic shocks, free boundary problems, elliptic-hyperbolic, nonlinear equations, second-order, mixed type, existence, uniqueness, stability, entropy solutions, Euler equations, compressible flow. 
head and an angle smaller than a certain degree, the uniform outflow with supersonic speed may produce a nontransonic shock (hyperbolic-hyperbolic shock) attached to the sharp head; the existence and stability of such shocks can be found in 11, 12, 19, 22, 25, 29, 32] and the references cited therein.

Transonic shocks also arise in many other situations of physical importance. For example, when a plane shock hits a wedge head on, a self-similar pattern of regularly reflected shock travels outward as the shock moves forward in time, provided either the wedge angle is large or the strength of the plane shock is large; then some part or all of the reflected shock may form a transonic shock dividing two regions of smooth flow, which is hyperbolic outside the shock and elliptic inside it (see [18, 28]). Steady transonic shocks are also very useful for constructing global solutions for some time-dependent problems (see [10]).

In this paper, we prove the existence and stability of steady multidimensional transonic shocks (hyperbolic-elliptic shocks) for (1.1) under a $C^{2, \alpha}, \alpha \in(0,1)$, steady perturbation of the upstream supersonic flow. We consider (1.1) in a bounded domain $\Omega:=Q^{n-1} \times\left(-N_{1}, N_{2}\right)$ with $Q^{n-1}=(0, a)^{n-1}$, with Neumann boundary conditions $\partial_{\nu} u=0$ on $\partial Q^{n-1} \times\left(-N_{1}, N_{2}\right)$, and Dirichlet conditions on $x_{n}=-N_{1}$ and $x_{n}=N_{2}$; that is, a flow in a channel (with quadratic cross-section if $n=3$ ). Our results indicate that, for any given upstream supersonic flow $\varphi^{-}$which is sufficiently close in $C^{2, \alpha}$ to a uniform flow in the direction $x_{n}$, there exists a unique solution $\varphi$ of (1.1) with boundary data described above such that $\varphi=\varphi^{-}$in the supersonic region $\Omega^{-}$of $\varphi$, and equation (1.1) is elliptic in $\Omega^{+}:=\Omega \backslash \overline{\Omega^{-}}$. The shock surface $S$ dividing $\Omega^{+}$and $\Omega^{-}$is $C^{2, \alpha}$; that is, $S$ is a graph $x_{n}=f\left(x_{1}, \cdots, x_{n-1}\right)$ with $f \in C^{2, \alpha}$. The solution $\varphi$ is stable under the $C^{2, \alpha}$ steady perturbation of the supersonic flow $\varphi^{-}$.

The transonic shock problem can be formulated into a free boundary problem: The free boundary is the location of the transonic shock, and the free boundary condition is the Rankine-Hugoniot jump condition on the shock. The equation is hyperbolic in the upstream region where the given $C^{2, \alpha}$ perturbed flow is supersonic. We are looking for the location of free boundary such that the free boundary condition holds and equation (1.1) is elliptic in the downstream region.

In order to solve this free boundary problem, we first consider a one-phase problem for a uniformly elliptic equation obtained by a modification of (1.1) away from the elliptic region: A solution satisfies the modified equation in the downstream region and the modified free boundary condition and coincides with the given hyperbolic phase in the upstream region. Then, by a gradient estimate, we show that the solution in fact solves the original problem. In order to avoid the difficulties related to the study of the free boundary up to the fixed boundary, we use a reflection technique to extend the domain so that the whole free boundary lies in the interior of the extended domain.

One of the main difficulties of the modified free boundary problem (Problem C below) is that it does not directly fit into the variational framework in Alt-Caffarelli [1] and Alt-Caffarelli-Friedman [2]. Indeed, according to Remark 3.1] Problem C can be reformulated into the following form: Find a nonnegative solution $u \in C(\Omega)$ satisfying suitable boundary conditions on $\partial \Omega$ and

$$
\begin{array}{cl}
\operatorname{div} A(x, D u)=f(x) & \text { in } \Omega^{+}:=\{u>0\} \\
A(x, D u) \cdot \nu=G(x, \nu) & \text { on } S:=\partial \Omega^{+} \backslash \partial \Omega,
\end{array}
$$


where $\nu$ is the unit normal vector to $S$ towards the unknown phase. The equation is quasilinear, uniformly elliptic, while the dependence on $\nu$ in the function $G(x, \nu)$ has a certain structure. The methods of [1] and [2] are directly applicable if $A(x, D u)=a(x,|D u|) D u$, where $a(x, s)$ is a scalar function, and $G$ is independent of $\nu$. However, both conditions do not hold in our problem. On the other hand, the nonlinearity in our problem makes it difficult to apply the Harnack inequality approach of Caffarelli [6]. In particular, a boundary comparison principle for positive solutions of elliptic equations in Lipschitz domains is still unavailable in the case that nonlinear equations are not homogeneous with respect to $D^{2} u, D u$ and $u$, which is our case.

The approach we develop here is an iteration scheme, which is based on the nondegeneracy of the free boundary condition: The jump of the normal derivative of a solution across the free boundary has a strictly positive lower bound. The nondegeneracy is also essential in other approaches to free boundary problems, for example, see Alt-Caffarelli 1], Alt-Caffarelli-Friedman [2, 3], and Caffarelli [6]. In terms of the problem (1.5) and (1.6), the iteration process is the following: Let the domain $\Omega_{i}^{+}$be given so that $S_{i}:=\partial \Omega_{i}^{+} \backslash \partial \Omega$ is in $C^{2, \alpha}$. We solve the oblique derivative problem (1.5) and (1.6) in $\Omega_{i}^{+}$(where the fixed boundary conditions on $\partial \Omega_{i}^{+} \cap \partial \Omega$ are also used) to obtain the solution $u_{i} \in C^{2, \alpha}\left(\overline{\Omega_{i}^{+}}\right)$. However, $u_{i}$ is not identically zero on $S_{i}$ in general. Using the nondegeneracy and geometry of our problem, we extend $u_{i}$ to the whole domain $\Omega$ so that $u_{i} \in C^{2, \alpha}(\bar{\Omega})$ and $\partial_{x_{n}} u_{i} \geq a>0$ in $\Omega$. Thus, the level set $S_{i+1}:=\left\{u_{i}=0\right\} \cap \Omega$ is a $C^{2, \alpha}$ surface. We define $\Omega_{i+1}^{+}:=\{u>0\}$ for the next step. The fixed point $\Omega^{+}$of this process determines a solution of the free boundary problem, since the corresponding solution $u$ of (1.5) and (1.6) satisfies $u>0$ on $\Omega^{+}$and $u=0$ on $S$. On the other hand, since the right-hand side of the free boundary condition (1.6) depends on $\nu$, the elliptic estimates alone are not sufficient to get the existence of a fixed point. However, the structure of our problem allows us to obtain better estimates for the iteration and to prove the existence of a fixed point.

The uniqueness and stability of solutions of the free boundary problem are obtained by using the regularity and nondegeneracy of solutions.

The nonlinear approach we develop here can be applied for solving other multidimensional transonic shock problems. As a direct example, in Section 7, we establish the existence and stability of multidimensional transonic shocks near spherical or circular transonic shocks. Another advantage of this approach is that it can be applied to multidimensional free boundary problems with more general fixed boundary conditions. Furthermore, our approach and results in this paper can extend to the problems with a steady $C^{1, \alpha}, \alpha \in(0,1)$, perturbation of the upstream supersonic flow and/or the problem with unbounded domains (see Chen-Feldman [9]).

A similar problem was considered in Canić-Keyfitz-Lieberman 8 for the twodimensional transonic small-disturbance (TSD) equation, which governs the behavior of the first nontrivial term in the geometric optics expansion to (1.1) near a certain physical point. The TSD model can be written as a second-order nonlinear equation of mixed type in two dimensions with coefficients depending only upon the unknown function itself. The main difference between the TSD model and (1.1) is that the coefficients of (1.1) depend on the gradient of the unknown function, while the coefficients of the TSD equation are independent of the gradient of the unknown function that generates additional compactness on which the approach in 
[8] relies. For other related results, we refer the reader to Majda [26] on the existence and stability, locally in time, of multidimensional shock fronts for the Euler equations for compressible fluids.

In Section 2, we formulate the multidimensional transonic shock problem into a free boundary problem, and then we describe the main theorems of this paper. In Section 3, we introduce a subsonic truncation procedure and an extension procedure to reformulate the free boundary problem for the equation of mixed type into the free boundary problem for a second-order, nonlinear, uniformly elliptic equation with a nondegenerate free boundary condition and to resolve the difficulties for the study of the free boundary up to the fixed boundary. In Section 4, we introduce an iteration scheme and prove the existence of a fixed point, that is, the existence of a solution of the truncated free boundary problem. By choosing the $C^{2, \alpha}$ perturbation small in the hyperbolic region, we obtain an a priori gradient estimate to ensure that our solution is the solution of the original free boundary problem. We show the stability and uniqueness of the solution of the free boundary problem in Sections 5 and 6 . In Section 7, we give another application of our approach for establishing the existence and stability of multidimensional transonic shocks near spherical or circular transonic shocks.

\section{TransoniC SHOCKS, FREE BOUndARIES, AND MAIN THEOREMS}

In this section we formulate the multidimensional transonic shock problem into a free boundary problem for (1.1), and then we describe the main theorems of this paper.

A function $\varphi \in W^{1, \infty}(\Omega)$ is a weak solution of (1.1) if

(i) $|D \varphi(x)| \leq 1 / \sqrt{\theta} \quad$ a.e. $\quad x \in \Omega \quad$ (physical region);

(ii) for any $w \in C_{0}^{\infty}(\Omega)$,

$$
\int_{\Omega} \rho\left(|D \varphi|^{2}\right) D \varphi \cdot D w d x=0 .
$$

We are interested in the weak solutions with shocks. Let $\Omega^{+}$and $\Omega^{-}$be open nonempty subsets of $\Omega$ such that

$$
\Omega^{+} \cap \Omega^{-}=\emptyset, \quad \overline{\Omega^{+}} \cup \overline{\Omega^{-}}=\bar{\Omega},
$$

and $S=\partial \Omega^{+} \backslash \partial \Omega$. Let $\varphi \in W^{1, \infty}(\Omega)$ be a weak solution of (1.1) so that $\varphi \in$ $C^{2}\left(\Omega^{ \pm}\right) \cap C^{1}\left(\overline{\Omega^{ \pm}}\right)$and $D \varphi$ has a jump across $S$.

We first derive the conditions on $S$ which is an $(n-1)$-dimensional smooth surface. First, the requirement $\varphi \in W^{1, \infty}(\Omega)$ yields $\operatorname{curl}(D \varphi)=0$ in the sense of distributions, which implies

$$
\varphi_{\tau}^{+}=\varphi_{\tau}^{-} \quad \text { on } S
$$

where

$$
\varphi_{\tau}^{ \pm}:=D \varphi^{ \pm}-\left(D \varphi^{ \pm} \cdot \nu\right) \nu
$$

are the tangential gradients of $\varphi$ on $S$ in the tangential space with $(n-1)$-dimension on the $\Omega^{ \pm}$sides, respectively, and $\nu$ is the unit normal vector to $S$ from $\Omega^{-}$to $\Omega^{+}$. Then we simply write $\varphi_{\tau}:=\varphi_{\tau}^{ \pm}$on $S$ and assume

$$
\varphi^{+}=\varphi^{-} \quad \text { on } S .
$$


Now, for $w \in C_{0}^{\infty}(\Omega)$, we use (1.1) and (2.1) to compute

$$
\begin{aligned}
0 & =\int_{\Omega} \rho\left(|D \varphi|^{2}\right) D \varphi \cdot D w d x \\
& =\left(\int_{\Omega^{+}}+\int_{\Omega^{-}}\right) \rho\left(|D \varphi|^{2}\right) D \varphi \cdot D w d x \\
& =-\int_{\partial \Omega^{+}} \rho\left(|D \varphi|^{2}\right) D \varphi \cdot \nu w d \mathcal{H}^{n-1}+\int_{\partial \Omega^{-}} \rho\left(|D \varphi|^{2}\right) D \varphi \cdot \nu w d \mathcal{H}^{n-1} \\
& =\int_{S}\left(-\rho\left(\left|D \varphi^{+}\right|^{2}\right) D \varphi^{+} \cdot \nu+\rho\left(\left|D \varphi^{-}\right|^{2}\right) D \varphi^{-} \cdot \nu\right) w d \mathcal{H}^{n-1} .
\end{aligned}
$$

Thus, another condition on $S$, which measures the jump of the normal derivative of $\varphi$ across $S$, is

$$
\left[\rho\left(|D \varphi|^{2}\right) D \varphi \cdot \nu\right]_{S}=0
$$

where the bracket denotes the difference between the values of the function along $S$ on the $\Omega^{+}$and $\Omega^{-}$sides, respectively. That is,

$$
\rho\left(\left|D \varphi^{+}\right|^{2}\right) \varphi_{\nu}^{+}=\rho\left(\left|D \varphi^{-}\right|^{2}\right) \varphi_{\nu}^{-} \quad \text { on } S,
$$

where $\varphi_{\nu}^{ \pm}=D \varphi^{ \pm} \cdot \nu$ are the values of the normal derivative of $\varphi$ on the $\Omega^{ \pm}$sides, and

$$
\rho\left(\left|D \varphi^{ \pm}\right|^{2}\right)=\left(1-\theta\left|\varphi_{\tau}^{ \pm}\right|^{2}-\theta\left|\varphi_{\nu}^{ \pm}\right|^{2}\right)^{\frac{1}{2 \theta}}
$$

respectively.

Lemma 2.1. Let $K>0$. Then the function

$$
\Phi_{K}(p):=\left(K-\theta p^{2}\right)^{\frac{1}{2 \theta}} p,
$$

defined for $p \in[0, \sqrt{K / \theta}]$, satisfies

(i) $\lim _{p \rightarrow 0} \Phi_{K}(p)=\lim _{p \rightarrow \sqrt{K / \theta}} \Phi_{K}(p)=0$;

(ii) $\Phi_{K}(p)>0$ for $p \in(0, \sqrt{K / \theta})$;

(iii) $0<\Phi_{K}^{\prime}(p) \leq K^{\frac{1}{2 \theta}}$ for $p \in\left(0, p_{\text {sonic }}^{K}\right)$, and $\Phi_{K}^{\prime}(p)<0$ for $p \in\left(p_{\text {sonic }}^{K}, \sqrt{K / \theta}\right) ;$

(iv) $\Phi_{K}^{\prime \prime}(p)<0$ for $p \in\left(0, p_{\text {sonic }}^{K}\right]$,

where

$$
p_{\text {sonic }}^{K}:=\sqrt{K /(\theta+1)} .
$$

Proof. Properties (i) and (ii) are obvious. For $p \in(0, \sqrt{K / \theta})$, we compute

$$
\begin{aligned}
& \Phi_{K}^{\prime}(p)=\left(K-\theta p^{2}\right)^{\frac{1}{2 \theta}-1}\left(K-(\theta+1) p^{2}\right), \\
& \Phi_{K}^{\prime \prime}(p)=p\left(K-\theta p^{2}\right)^{\frac{1}{2 \theta}-2}\left((\theta+1) p^{2}-3 K\right),
\end{aligned}
$$

and note that $p_{\text {sonic }}^{K}=\sqrt{K /(\theta+1)} \in(0, \sqrt{K / \theta})$. Then (iii) and (iv) follow. 
Suppose that $\varphi$ is a solution satisfying

$$
|D \varphi|<p_{\text {sonic }}^{1}:=1 / \sqrt{\theta+1} \quad \text { in } \Omega^{+}, \quad|D \varphi|>p_{\text {sonic }}^{1} \text { in } \Omega^{-},
$$

and

$$
D \varphi^{ \pm} \cdot \nu>0 \text { on } S,
$$

besides (2.2) and (2.4). Then $\varphi$ is a transonic shock solution with transonic shock $S$, which divides the subsonic region $\Omega^{+}$and the supersonic region $\Omega^{-}$. In addition, $\varphi$ satisfies the physical entropy condition (see Courant-Friedrichs [13]; also see Lax [21]):

$$
\rho\left(\left|D \varphi^{-}\right|^{2}\right)<\rho\left(\left|D \varphi^{+}\right|^{2}\right)
$$

which implies, by (2.8), that the density $\rho$ increases in the flow direction.

Note that equation (1.1) is elliptic in the subsonic region $\Omega^{+}$and hyperbolic in the supersonic region $\Omega^{-}$.

Let $\left(x^{\prime}, x_{n}\right)$ be the coordinates of $\mathbf{R}^{n}$ with $x_{n} \in \mathbf{R}$ and $x^{\prime}=\left(x_{1}, \cdots, x_{n-1}\right) \in$ $\mathbf{R}^{n-1}$. From now on, we denote $\Omega:=(0, a)^{n-1} \times\left(-N_{1}, N_{2}\right)$.

Let $q^{-} \in\left(p_{\text {sonic }}^{1}, 1 / \sqrt{\theta}\right)$ and $\varphi_{0}^{-}(x):=q^{-} x_{n}$. Then $\varphi_{0}^{-}$is a supersonic solution in $\Omega$. According to Lemma 2.1 there exists a unique $q^{+} \in\left(0, p_{\text {sonic }}^{1}\right)$ such that

$$
\left(1-\theta\left(q^{+}\right)^{2}\right)^{\frac{1}{2 \theta}} q^{+}=\left(1-\theta\left(q^{-}\right)^{2}\right)^{\frac{1}{2 \theta}} q^{-} .
$$

Define $\varphi_{0}^{+}(x):=q^{+} x_{n}$ in $\Omega$. Then the function

$$
\varphi_{0}(x)=\min \left(\varphi_{0}^{+}(x), \varphi_{0}^{-}(x)\right) \text { for } x \in \Omega
$$

is a transonic shock solution in $\Omega$, in which $\Omega_{0}^{ \pm}=\left\{ \pm x_{n}>0\right\} \cap \Omega$ are the subsonic and supersonic regions of $\varphi_{0}$, respectively. Also note that, on $\partial(0, a)^{n-1} \times$ $\left(-N_{1}, N_{2}\right)$, the boundary condition $\left(\varphi_{0}\right)_{\nu}=0$ holds.

We study perturbations of the solution (2.11). We use the following Hölder norms: For $\alpha \in(0,1)$ and any nonnegative integer $k$,

$$
\begin{aligned}
& {[u]_{k, 0, \Omega}=\sum_{|\beta|=k} \sup _{x \in \Omega}\left|D^{\beta} u(x)\right|,} \\
& {[u]_{k, \alpha, \Omega}=\sum_{|\beta|=k} \sup _{x, y \in \Omega, x \neq y} \frac{\left|D^{\beta} u(x)-D^{\beta} u(y)\right|}{|x-y|^{\alpha}},} \\
& \|u\|_{k, 0, \Omega}=\sum_{j=0}^{k}[u]_{j, 0, \Omega}, \quad\|u\|_{k, \alpha, \Omega}=\|u\|_{k, 0, \Omega}+[u]_{k, \alpha, \Omega},
\end{aligned}
$$

where $\beta=\left(\beta_{1}, \cdots, \beta_{n}\right), \beta_{l} \geq 0$ integers, $D^{\beta}=\partial_{x_{1}}^{\beta_{1}} \cdots \partial_{x_{n}}^{\beta_{n}}$, and $|\beta|=\beta_{1}+\cdots+\beta_{n}$.

Our transonic shock problem is the following.

Problem A. Given a supersonic solution $\varphi^{-}$of (1.1) in $\Omega$, which is a $C^{2, \alpha}$ perturbation of $\varphi_{0}^{-}$, for some $\alpha>0$ :

$$
\left\|\varphi^{-}-\varphi_{0}^{-}\right\|_{2, \alpha, \Omega} \leq \sigma,
$$

with $\sigma>0$ small, and satisfies

$$
\varphi_{\nu}^{-}=0 \quad \text { on } \partial(0, a)^{n-1} \times\left(-N_{1}, N_{2}\right),
$$

find a transonic shock solution $\varphi$ in $\Omega$ such that

$$
\varphi=\varphi^{-} \quad \text { in } \Omega^{-},
$$


where $\Omega^{-}$is the supersonic region of $\varphi$ in $\Omega$, defined by $\Omega^{-}:=\Omega \backslash \overline{\Omega^{+}}$ with $\Omega^{+}:=\left\{x \in \Omega:|D \varphi(x)|<p_{\text {sonic }}^{1}\right\}$ which is the subsonic region of $\varphi$, and

$$
\begin{array}{lll}
\varphi=\varphi^{-}, \varphi_{x_{n}}=\varphi_{x_{n}}^{-} & \text {on } & (0, a)^{n-1} \times\left\{-N_{1}\right\}, \\
\varphi=\varphi_{0}^{+} & \text {on } & (0, a)^{n-1} \times\left\{N_{2}\right\}, \\
\varphi_{\nu}=0 & \text { on } & \partial(0, a)^{n-1} \times\left(-N_{1}, N_{2}\right) .
\end{array}
$$

In order to construct a solution of Problem A, we reformulate it into a more general free boundary problem for the subsonic part of the solution. The following heuristic observation motivates our formulation: Since $\varphi=\varphi^{-}$in $\Omega^{-},|D \varphi|<p_{\text {sonic }}^{1}<\left|D \varphi^{-}\right|$ in $\Omega^{+},\left|D \varphi^{-}\right| \sim \partial_{x_{n}} \varphi^{-}>p_{\text {sonic }}^{1}$ in $\Omega$, and we expect that $\Omega^{+}=\left\{x_{n}>f\left(x^{\prime}\right)\right\} \cap \Omega$ and $|D \varphi| \sim \partial_{x_{n}} \varphi<p_{\text {sonic }}^{1}$ in $\Omega^{+}$with [2.3) across the free boundary, then $\varphi$ should satisfy

$$
\varphi(x) \leq \varphi^{-}(x) \text { for } x \in \Omega .
$$

Now we can formulate the following free boundary problem:

Problem B. Find $\varphi \in C(\bar{\Omega})$ such that

(i) $\varphi$ satisfies (2.16) in $\Omega$ and (2.15) on $\partial \Omega$;

(ii) $\varphi \in C^{2, \alpha}\left(\overline{\Omega^{+}}\right)$is a solution of (1.1) in $\Omega^{+}:=\{x \in \Omega: \varphi(x)<$ $\left.\varphi^{-}(x)\right\}$, the noncoincidence set;

(iii) the free boundary $S=\partial \Omega^{+} \cap \Omega$ is given by the equation $x_{n}=$ $f\left(x^{\prime}\right)$ for $x^{\prime} \in(0, a)^{n-1}$ so that $\Omega^{+}=\left\{x_{n}>f\left(x^{\prime}\right)\right\} \cap \Omega$, where $f \in C^{2, \alpha}\left([0, a]^{n-1}\right)$;

(iv) the free boundary condition (2.4) holds on $S$.

Note that the definitions of the regions $\Omega^{ \pm}$in Problems A and B are a priori different, since the formula of $\Omega^{+}$given in the formulation of Problem B is a new definition, rather than an expression of the region $\Omega^{+}$defined in Problem $\mathrm{A}$ above. In particular, in the free boundary problem (Problem B), we do not require that the phase $\varphi^{-}$be a solution of (1.1) and that $\varphi$ be subsonic in $\Omega^{+}$, although we require it in Problem A so that the free boundary is a transonic shock.

We will show that, if the perturbation $\varphi^{-}-\varphi_{0}^{-}$is small enough in $C^{2, \alpha}$, then the free boundary problem (Problem B) has a solution which is a transonic shock solution to Problem A. Furthermore, the transonic shock is stable under any small $C^{2, \alpha}$ perturbation of $\varphi^{-}$. Precisely, we have the following theorem.

Theorem 2.1. Let $q^{+} \in\left(0, p_{\text {sonic }}^{1}\right)$ and $q^{-} \in\left(p_{\text {sonic }}^{1}, 1 / \sqrt{\theta}\right)$ satisfy (2.10), and let $\varphi_{0}$ be the transonic shock solution (2.11). Then there exist positive constants $\sigma_{0}$, $C_{1}$, and $C_{2}$ depending only on $n, \gamma, q^{+}$, and $\Omega$ such that, for every $\sigma \leq \sigma_{0}$ and any supersonic solution $\varphi^{-}$of (1.1) satisfying the conditions stated in Problem A, there exists a unique solution $\varphi$ of Problem A satisfying

$$
\left\|\varphi-\varphi_{0}^{+}\right\|_{2, \alpha, \Omega^{+}} \leq C_{1} \sigma .
$$

In addition, $\Omega^{+}=\left\{x_{n}>f\left(x^{\prime}\right)\right\} \cap \Omega$ where $f: \mathbf{R}^{n-1} \rightarrow \mathbf{R}$ satisfies

$$
\begin{aligned}
& \|f\|_{2, \alpha, \mathbf{R}^{n-1}} \leq C_{2} \sigma, \\
& D_{x^{\prime}} f=0 \quad \text { on } \partial(0, a)^{n-1},
\end{aligned}
$$

that is, the shock surface $S=\left\{\left(x^{\prime}, x_{n}\right): x_{n}=f\left(x^{\prime}\right), x^{\prime} \in \mathbf{R}^{n-1}\right\} \cap \Omega$ is in $C^{2, \alpha}$ and $S$ is orthogonal to $\partial \Omega$ at their intersection points. 
Theorem 2.1 is a corollary of the following corresponding theorem for Problem B.

Theorem 2.2. Let $q^{+}$and $q^{-}$be as in Theorem 2.1 Then there exist positive constants $\sigma_{0}, C_{1}$, and $C_{2}$ depending only on $n, \gamma, q^{+}$, and $\Omega$ such that, for every $\sigma \leq \sigma_{0}$ and any function $\varphi^{-}$satisfying (2.13) and (2.14), there exists a unique solution $\varphi$ of Problem $B$ satisfying

$$
\left\|\varphi-\varphi_{0}^{+}\right\|_{2, \alpha, \Omega^{+}} \leq C_{1} \sigma .
$$

In addition, $\Omega^{+}=\left\{x_{n}>f\left(x^{\prime}\right)\right\} \cap \Omega$ where $f: \mathbf{R}^{n-1} \rightarrow \mathbf{R}$ satisfies

$$
\begin{aligned}
& \|f\|_{2, \alpha, \mathbf{R}^{n-1}} \leq C_{2} \sigma, \\
& D_{x^{\prime}} f=0 \text { on } \partial(0, a)^{n-1} .
\end{aligned}
$$

Remark 2.1. If the hyperbolic phase is $C^{\infty}$, then the solution and the corresponding free boundary in Theorem 2.2 are also $C^{\infty}$. Furthermore, our results can extend to the problem with a steady $C^{1, \alpha}, \alpha \in(0,1)$, perturbation of the upstream supersonic flow and/or general Dirichlet data $h\left(x^{\prime}\right), x^{\prime} \in \mathbf{R}^{n-1}$, at $x_{n}=N_{2}$ satisfying

$$
\left\|h-\varphi_{0}^{+}\right\|_{1, \alpha, \mathbf{R}^{n-1}} \leq C \sigma
$$

Also, the Dirichlet data in Problem B may be replaced by the corresponding Neumann data satisfying the global solvability condition. Furthermore, the bounded domain in the problem can be replaced by unbounded domains, especially the unbounded cylinder up to $x_{n}=\infty$. See Chen-Feldman [9] for the details.

The following features of equation (1.1) and the free boundary condition (2.5) will be employed in the proof of Theorems 2.1 and 2.2. The nonlinear equation (1.1) is uniformly elliptic only if $|D \varphi|<p_{\text {sonic }}^{1}-\varepsilon$ in $\Omega^{+}$for some $\varepsilon>0$; the quantity $\left|D \varphi^{+}\right|=\left(\left(\varphi_{\nu}^{+}\right)^{2}+\left|\varphi_{\tau}\right|^{2}\right)^{1 / 2}$ on $S$ is subsonic only if $\varphi_{\tau}$ is sufficiently small; and the free boundary condition (2.5) is uniformly nondegenerate (i.e., $\varphi_{\nu}^{-}-\varphi_{\nu}^{+}$is bounded from below by a positive constant on $S$ ) only if $\varphi_{\nu}^{-}>p_{\text {sonic }}^{K}+\varepsilon$ on $S$ for some $\epsilon>0$ with $K=1-\theta\left|\varphi_{\tau}\right|^{2}$. By (2.13), these conditions hold if, for any $x \in S$, the unit normal vector $\nu(x)$ to $S$ is sufficiently close to being orthogonal to $\left\{x_{n}=0\right\}$.

To establish Theorem 2.2 (hence Theorem 2.1), we first introduce and solve a truncated problem, by modifying both the nonlinear equation (1.1) and the free boundary condition (2.5), in order to make the equation uniformly elliptic and the free boundary condition nondegenerate. Then, for small $\sigma$, a gradient bound for the solution implies that it indeed solves the original free boundary problem, Problem B, hence Problem A.

\section{Subsonic TRUnCATIONS}

In this section, we introduce a truncated free boundary problem, by modifying both the nonlinear equation (1.1) and the free boundary condition (2.5), to make the equation uniformly elliptic and the free boundary condition nondegenerate; we also extend the domain $\Omega$ to the domain $\Omega_{e}$ to overcome the difficulties for the study of the free boundary up to the fixed boundary for Problem B. 
3.1. Truncation of equation (1.1). The truncation procedure below is motivated by the argument in [3, pp. 87-90].

First, we note that the ellipticity condition for (1.1) at $|D \varphi|=q$ is (1.3), which is equivalent to

$$
\Phi_{1}^{\prime}(q)>0,
$$

where $\Phi_{K}(p)$ is the function introduced in Lemma 2.1 .

By Lemma 2.1(iii), the inequality (3.1) holds for $q \in\left(0, p_{\text {sonic }}^{1}\right)$. We modify the function $\Phi_{1}(q)$ so that the new function $\tilde{\Phi}_{1}(q)$ satisfies (3.1) uniformly for all $q>0$ and, around $q^{+}$, the function $\tilde{\Phi}_{1}(q)=\Phi_{1}(q)$.

Let

$$
\varepsilon=\frac{p_{\text {sonic }}^{1}-q^{+}}{2}
$$

Let $y=c_{0} q+c_{1}$ be the tangent line of the graph of $y=\Phi_{1}(q)$ at $q=p_{\text {sonic }}^{1}-\varepsilon$. Then, using Lemma 2.1(1iii), we obtain

$$
c_{0}=\Phi_{1}^{\prime}\left(p_{\text {sonic }}^{1}-\varepsilon\right)>0 .
$$

Now the function $\tilde{\Phi}_{1}:[0, \infty) \rightarrow \mathbf{R}$, defined by

$$
\tilde{\Phi}_{1}(q)= \begin{cases}\Phi_{1}(q) & \text { if } \quad 0 \leq q<p_{\text {sonic }}^{1}-\varepsilon \\ c_{0} q+c_{1} & \text { if } \quad q>p_{\text {sonic }}^{1}-\varepsilon\end{cases}
$$

satisfies $\tilde{\Phi}_{1} \in C^{1,1}([0, \infty))$.

Define

$$
\tilde{\rho}\left(q^{2}\right)=\frac{\tilde{\Phi}_{1}(q)}{q} \quad \text { for } q \in[0, \infty),
$$

that is,

$$
\tilde{\rho}(s)= \begin{cases}\rho(s) & \text { if } 0 \leq s<\left(p_{\text {sonic }}^{1}-\varepsilon\right)^{2}, \\ c_{0}+\frac{c_{1}}{\sqrt{s}} & \text { if } s>\left(p_{\text {sonic }}^{1}-\varepsilon\right)^{2} .\end{cases}
$$

Then $\tilde{\rho} \in C^{1,1}([0, \infty))$, and

$$
\tilde{\rho}\left(q^{2}\right)=\rho\left(q^{2}\right) \quad \text { if } 0 \leq q<p_{\text {sonic }}^{1}-\varepsilon .
$$

By Lemma 2.1 (iii)-(iv) and the definition (3.3) of $\tilde{\Phi}_{1}$,

$$
0<c_{0} \leq \tilde{\Phi}_{1}^{\prime}(q)=\tilde{\rho}\left(q^{2}\right)+2 q^{2} \tilde{\rho}^{\prime}\left(q^{2}\right) \leq C, \quad \text { for } q \in(0, \infty),
$$

for some constant $C>0$. Thus, the equation

$$
\tilde{\mathcal{L}} \varphi:=\operatorname{div}\left(\tilde{\rho}\left(|D \varphi|^{2}\right) D \varphi\right)=0
$$

is uniformly elliptic, with ellipticity constants depending only on $q^{+}$and $\gamma$.

We also perform the corresponding truncation of the free boundary condition $(2.5)$ :

$$
\tilde{\rho}\left(|D \varphi|^{2}\right) \varphi_{\nu}=\rho\left(\left|D \varphi^{-}\right|^{2}\right) D \varphi^{-} \cdot \nu \quad \text { on } S .
$$

On the right-hand side of (3.7), we use the nontruncated function $\rho$ since $\rho \neq \tilde{\rho}$ on the range of $\left|D \varphi^{-}\right|^{2}$. Note that (3.7), with the right-hand side considered as a known function, is the conormal boundary condition for the uniformly elliptic equation (3.6).

Thus, we solve the following free boundary problem, which is a truncated version of Problem B. 
Problem C. Find $\varphi \in C(\bar{\Omega})$ such that

(i) $\varphi$ satisfies (2.16) in $\Omega$ and (2.15) on $\partial \Omega$;

(ii) $\varphi \in C^{2, \alpha}\left(\overline{\Omega^{+}}\right)$is a solution of [3.6) in $\Omega^{+}:=\left\{\varphi(x)<\varphi^{-}(x)\right\} \cap \Omega$, the noncoincidence set;

(iii) the free boundary $S=\partial \Omega^{+} \cap \Omega$ is given by the equation $x_{n}=f\left(x^{\prime}\right)$ for $x^{\prime} \in(0, a)^{n-1}$ so that $\Omega^{+}=\left\{x_{n}>f\left(x^{\prime}\right)\right\} \cap \Omega$, where $f \in C^{2, \alpha}\left([0, a]^{n-1}\right)$ and $D_{x^{\prime}} f=0$ on $\partial\left((0, a)^{n-1} \times\left(-N_{1}, N_{2}\right)\right)$;

(iv) the free boundary condition (3.7) holds on $S$.

Remark 3.1. Introduce the function

$$
u:=\varphi^{-}-\varphi
$$

and rewrite Problem $\mathrm{C}$ in terms of the function $u(x)$. Then, by (2.16), the problem is to find a nonnegative $u \in C(\bar{\Omega})$, with boundary conditions determined by (2.15) and $\varphi^{-}$, satisfying (1.5) and (1.6) with

$$
\begin{aligned}
& A(x, P)=\tilde{\rho}\left(\left|D \varphi^{-}(x)-P\right|^{2}\right)\left(D \varphi^{-}(x)-P\right)-\tilde{\rho}\left(\left|D \varphi^{-}(x)\right|^{2}\right) D \varphi^{-}(x), \quad P \in \mathbf{R}^{n}, \\
& f(x)=-\operatorname{div}\left(\tilde{\rho}\left(\left|D \varphi^{-}(x)\right|^{2}\right) D \varphi^{-}(x)\right), \\
& G(x, \nu)=\left(\rho\left(\left|D \varphi^{-}(x)\right|^{2}\right)-\tilde{\rho}\left(\left|D \varphi^{-}(x)\right|^{2}\right)\right) D \varphi^{-}(x) \cdot \nu .
\end{aligned}
$$

3.2. Extension to the domain $\Omega_{e}=\mathbf{T}^{n-1} \times\left(-N_{1}, N_{2}\right)$. We now extend the domain $\Omega$ of the free boundary problem to the domain $\Omega_{e}$ to overcome the difficulties for the study of the free boundary up to the fixed boundary.

Observe that, if a function $\phi \in C^{2, \alpha}(\bar{\Omega})$ with $\Omega:=(0, a)^{n-1} \times\left(-N_{1}, N_{2}\right)$ and

$$
\phi_{\nu}=0 \quad \text { on } \partial(0, a)^{n-1} \times\left(-N_{1}, N_{2}\right),
$$

then $\phi$ can be extended to $\mathbf{R}^{n-1} \times\left[-N_{1}, N_{2}\right]$ so that the extension (still denoted) $\phi$ satisfies

$$
\phi \in C^{2, \alpha}\left(\mathbf{R}^{n-1} \times\left[-N_{1}, N_{2}\right]\right),
$$

and, for every $m=1, \cdots, n-1$ and $k=0, \pm 1, \pm 2, \cdots$,

$$
\phi\left(x_{1}, \cdots, x_{m-1}, k a-z, x_{m+1}, \cdots, x_{n}\right)=\phi\left(x_{1}, \cdots, x_{m-1}, k a+z, x_{m+1}, \cdots, x_{n}\right),
$$

that is, $\phi$ is symmetric with respect to every hyperplane $\left\{x_{m}=k a\right\}$. Indeed, for $\mathbf{k}=\left(k_{1}, \cdots, k_{n-1}, 0\right)$ with $k_{1}, \cdots, k_{n-1}$ integers, we define

$$
\phi(x+a \mathbf{k})=\phi\left(\eta\left(x_{1}, k_{1}\right), \cdots, \eta\left(x_{n-1}, k_{n-1}\right), x_{n}\right) \quad \text { for } x \in(0, a)^{n-1} \times\left[-N_{1}, N_{2}\right],
$$

with

$$
\eta(t, k)= \begin{cases}t & \text { if } k \text { is even, } \\ a-t & \text { if } k \text { is odd. }\end{cases}
$$

It follows from (3.9) that $\phi\left(x^{\prime}, x_{n}\right)$ is $2 a$-periodic in the directions $x_{1}, \cdots, x_{n-1}$ :

$$
\phi\left(x+2 a e_{m}\right)=\phi(x), \quad \text { for } x \in \mathbf{R}^{n-1} \times\left[-N_{1}, N_{2}\right], m=1, \cdots, n-1,
$$

where $e_{m}$ is the unit vector in the direction of $x_{m}$.

Thus, with respect to this $2 a$-periodicity, we can consider $\phi$ as a function on $\Omega_{e}:=\mathbf{T}^{n-1} \times\left[-N_{1}, N_{2}\right]$, where $\mathbf{T}^{n-1}$ is an $(n-1)$-dimensional flat torus with its coordinates given by the cube $(0,2 a)^{n-1}$. Note that (3.9) represents an extra symmetry condition, in addition to $\phi \in C^{2, \alpha}\left(\mathbf{T}^{n-1} \times\left[-N_{1}, N_{2}\right]\right)$, and (3.9) implies (3.8). 
Thus we can extend $\varphi^{-}$in this way, that is, $\varphi^{-} \in C^{2, \alpha}\left(\Omega_{e}\right)$ satisfies ([3.9). Also, $\varphi_{0}^{ \pm}$can be considered as the functions in $\Omega_{e}$ satisfying (3.9), since $\varphi_{0}^{ \pm}(x)=q^{ \pm} x_{n}$ in $\mathbf{R}^{n-1} \times\left[-N_{1}, N_{2}\right]$, which are independent of $x^{\prime}$.

Then we focus on the free boundary problem, Problem $\mathrm{C}$, on $\Omega_{e}$.

\section{Existence OF SOlutions}

In this section, we develop a nonlinear approach to prove the existence of solutions of the free boundary problem. Our approach is an iteration scheme, which is based on the nondegeneracy of the free boundary condition: The jump of the normal derivative of solutions across the free boundary has a strict lower bound. The iteration procedure in 4.1 has no additional compactness effect, which is different from that in 8 ; the elliptic estimates in $\$ 4.2$ alone do not produce what we require to get the existence of a fixed point. We use certain cancellations in order to get (4.20) and thus to close the argument for the existence of a fixed point.

4.1. Iteration procedure. Let $M \geq 1$. We set

$$
\mathcal{K}_{M}:=\left\{\psi \in C^{2, \alpha}\left(\overline{\Omega_{e}}\right):\left\|\psi-\varphi_{0}^{+}\right\|_{2, \alpha, \Omega_{e}} \leq M \sigma, \psi \text { satisfies (3.9) }\right\},
$$

where $\varphi_{0}^{+}(x)=q^{+} x_{n}$. According to the definition, $\mathcal{K}_{M}$ is convex and compact in $C^{2, \beta}\left(\overline{\Omega_{e}}\right), 0<\beta<\alpha$.

Let $\psi \in \mathcal{K}_{M}$. Since $q^{-}>q^{+}$, it follows that, if

$$
\sigma \leq \frac{q^{-}-q^{+}}{C(M+1)}
$$

with large $C$ depending only on $n$, then (4.1) and 2.13) imply

$$
\left(\varphi^{-}-\psi\right)_{x_{n}}(x) \geq \frac{q^{-}-q^{+}}{2}>0 .
$$

Then, by the implicit function theorem, the set $\Omega^{+}(\psi):=\left\{\psi(x)<\varphi^{-}(x)\right\} \cap \Omega_{e}$ has the form:

$$
\Omega^{+}(\psi)=\left\{x_{n}>f\left(x^{\prime}\right)\right\} \cap \Omega_{e}, \quad\|f\|_{2, \alpha, \mathbf{T}^{n-1}} \leq C M \sigma,
$$

with $C$ depending upon $q^{-}-q^{+}$. The corresponding unit normal vector

$$
\nu\left(x^{\prime}\right)=\frac{\left(-D_{x^{\prime}} f\left(x^{\prime}\right), 1\right)}{\sqrt{1+\left|D_{x^{\prime}} f\left(x^{\prime}\right)\right|^{2}}} \in C^{1, \alpha}\left(\mathbf{T}^{n-1} ; \mathbf{S}^{n-1}\right),
$$

and

$$
\left\|\nu-\nu_{0}\right\|_{1, \alpha, \mathbf{R}^{n-1}} \leq C M \sigma
$$

where $\nu_{0}$ is defined by

$$
\nu_{0}:=\frac{D \varphi_{0}^{+}}{\left|D \varphi_{0}^{+}\right|}=(0, \cdots, 0,1)^{\top} .
$$

Also, $\nu(\cdot)$ can be considered as a function on $S_{\psi}:=\left\{x_{n}=f\left(x^{\prime}\right)\right\}$. From the definition of $f\left(x^{\prime}\right)$, it follows that, for $x \in S_{\psi}$,

$$
\nu(x)=\frac{D \varphi^{-}(x)-D \psi(x)}{\left|D \varphi^{-}(x)-D \psi(x)\right|} .
$$

By the definition of $\mathcal{K}_{M}$ and (4.2), the formula (4.7) defines $\nu$ on $\Omega_{e}$, and

$$
\left\|\nu-\nu_{0}\right\|_{1, \alpha, \Omega_{e}} \leq C M \sigma, \quad C=C\left(q^{+}, q^{-}\right)
$$


Motivated by the free boundary condition (3.7), we define the function $G_{\psi}$ on $\Omega_{e}$ :

$$
G_{\psi}(x):=\rho\left(\left|D \varphi^{-}(x)\right|^{2}\right) D \varphi^{-}(x) \cdot \nu(x),
$$

where $\nu(\cdot)$ is defined by (4.7).

We now solve the following fixed-boundary value problem in the domain $\Omega^{+}(\psi)$ :

$$
\begin{aligned}
& \operatorname{div}\left(\tilde{\rho}\left(|D \varphi|^{2}\right) D \varphi\right)=0 \quad \text { in } \Omega^{+}(\psi), \\
& \tilde{\rho}\left(|D \varphi|^{2}\right) \varphi_{\nu}=G_{\psi} \quad \text { on } S_{\psi}, \\
& \varphi=N_{2} q^{+} \quad \text { on }\left\{x_{n}=N_{2}\right\}=\partial \Omega^{+}(\psi) \backslash S_{\psi},
\end{aligned}
$$

and we show that the solution $\varphi$ can be extended to the whole domain $\Omega_{e}$ so that $\varphi \in \mathcal{K}_{M}$.

4.2. Existence and uniqueness of the solution for the fixed boundary value problem (4.10)-(4.12). Now we show the existence and uniqueness of the solution $\varphi$ for the problem (4.10)-(4.12) and show that $\varphi$ is close in $C^{2, \alpha}\left(\overline{\Omega^{+}(\psi)}\right)$ to the unperturbed subsonic solution $\varphi_{0}^{+}$.

Proposition 4.1. Let $M \geq 1$. There exists $\sigma_{0}>0$, depending only on $n, \gamma, q^{+}$, $\Omega$, and $M$ such that, if $\sigma \in\left(0, \sigma_{0}\right)$ so that $\varphi^{-}$satisfies (2.13) and $\psi \in \mathcal{K}_{M}$, then there exists a unique solution $\varphi \in C^{2, \alpha}\left(\overline{\Omega^{+}(\psi)}\right)$ of the problem (4.10) -(4.12) that satisfies (3.9) and

$$
\left\|\varphi-\varphi_{0}^{+}\right\|_{2, \alpha, \Omega^{+}(\psi)} \leq C \sigma
$$

where $C$ depends only on $n, \gamma, q^{+}$, and $\Omega$, and is independent of $M, \psi \in \mathcal{K}_{M}$, and $\sigma \in\left(0, \sigma_{0}\right)$.

Proof. In the argument below, the constants $C$ and $C_{1}$ depend only on $n, \gamma, q^{+}$, and $\Omega$, and are independent of $M, \psi \in \mathcal{K}_{M}$, and $\sigma \in\left(0, \sigma_{0}\right)$, unless other dependence is specified. We divide the proof into four steps.

Step 1. We first rewrite the problem 4.10-4.12) in terms of the function $v:=\varphi-\varphi_{0}^{+}$. The problem then takes the form:

$$
\begin{aligned}
& \operatorname{div} A(D v)=0 \quad \text { in } \Omega^{+}(\psi), \\
& A(D v) \cdot \nu=g_{\psi} \quad \text { on } S_{\psi}, \\
& v=0 \quad \text { on } \Gamma_{1}:=\mathbf{T}^{n-1} \times\left\{N_{2}\right\} \equiv \partial \Omega^{+}(\psi) \backslash S_{\psi},
\end{aligned}
$$

where

$$
\begin{aligned}
& A(P)=\tilde{\rho}\left(\left|P+q^{+} \nu_{0}\right|^{2}\right)\left(P+q^{+} \nu_{0}\right)-\rho\left(\left(q^{+}\right)^{2}\right) q^{+} \nu_{0} \quad \text { for } P \in \mathbf{R}^{n}, \\
& g_{\psi}(x)=G_{\psi}(x)-\rho\left(\left(q^{+}\right)^{2}\right) q^{+} \nu(x) \cdot \nu_{0} .
\end{aligned}
$$

Thus, $v(x)$ satisfies the uniformly elliptic equation with the same ellipticity constants as those in (3.6). Note that

$$
A(0)=0 .
$$

Now we show the crucial (but simple) estimate of $g_{\psi}(x)$, based on a cancellation. We first note that

$$
g_{\psi}(x)=\left(\rho\left(\left|D \varphi^{-}(x)\right|^{2}\right) D \varphi^{-}(x)-\rho\left(\left|D \varphi_{0}^{+}(x)\right|^{2}\right) D \varphi_{0}^{+}(x)\right) \cdot \nu(x) .
$$

Furthermore,

$$
\rho\left(\left|D \varphi_{0}^{+}\right|^{2}\right) D \varphi_{0}^{+}=\rho\left(\left|D \varphi_{0}^{-}\right|^{2}\right) D \varphi_{0}^{-}
$$


in $\Omega_{e}$, since both sides of (4.19) are equal to $\rho\left(\left(q^{+}\right)^{2}\right) q^{+} \nu_{0}$. Thus,

$$
g_{\psi}(x)=\left(\rho\left(\left|D \varphi^{-}(x)\right|^{2}\right) D \varphi^{-}(x)-\rho\left(\left|D \varphi_{0}^{-}(x)\right|^{2}\right) D \varphi_{0}^{-}(x)\right) \cdot \nu(x) .
$$

Using (2.13) and (4.8), we obtain

$$
\left\|g_{\psi}\right\|_{1, \alpha, \Omega_{e}} \leq C\left\|D \varphi^{-}-D \varphi_{0}^{-}\right\|_{1, \alpha, \Omega_{e}}\|\nu\|_{1, \alpha, \Omega_{e}} \leq C \sigma(1+M \sigma) .
$$

Therefore, choosing $\sigma \leq \frac{1}{M}$, we have

$$
\left\|g_{\psi}\right\|_{1, \alpha, \Omega_{e}} \leq C \sigma .
$$

Step 2. In order to study the problem (4.14 -4.16), we now consider the corresponding linear problem:

$$
\left\{\begin{array}{l}
\overline{\mathcal{L}}[u]:=\sum_{i, j=1}^{n} a_{i j}(x) u_{x_{i} x_{j}}=r(x) \quad \text { in } \Omega^{+}(\psi), \\
\sum_{i=1}^{n} b_{i}(x) u_{x_{i}}=g(x) \quad \text { on } S_{\psi}, \\
u=0 \quad \text { on } \Gamma_{1} .
\end{array}\right.
$$

Here $a_{i j} \in C^{\alpha}\left(\overline{\Omega_{e}}\right)$ satisfy the ellipticity condition:

$$
\lambda|\xi|^{2} \leq \sum_{i, j=1}^{n} a_{i j}(x) \xi_{i} \xi_{j} \leq \Lambda|\xi|^{2} \quad \text { for any } \quad x \in \Omega_{e}, \xi \in \mathbf{R}^{n}
$$

with $0<\lambda<\Lambda$, and $b_{i} \in C^{1, \alpha}\left(\overline{\Omega_{e}}\right)$ satisfy

$$
\left|b_{i}(x)\right| \leq \Lambda
$$

and the strict obliqueness condition:

$$
b(x) \cdot \nu(x) \geq \kappa>0 \quad \text { for any } x \in S_{\psi},
$$

where $b(x)$ denotes the vector $\left(b_{1}, \cdots, b_{n}\right)(x)$.

Lemma 4.2. Let $M \geq 1$ and $\psi \in \mathcal{K}_{M}$. Then there exists $\sigma_{0}$ depending only on $n, \gamma, q^{+}, \Omega$, and $M$ such that, if $\sigma \in\left(0, \sigma_{0}\right)$ so that $\varphi^{-}$satisfy (2.13) and $u \in C^{1}\left(\overline{\Omega^{+}(\psi)}\right) \cap C^{2}\left(\Omega^{+}(\psi)\right)$ is a solution of (4.21) with $r \in C^{\alpha}\left(\overline{\Omega_{e}}\right)$, then

(i) there exists $C$ depending only on $\lambda, \Lambda, \kappa$, and $\Omega$ such that

$$
\|u\|_{0, \Omega^{+}(\psi)} \leq C\left(\|r\|_{0, \Omega^{+}(\psi)}+\|g\|_{0, \Omega^{+}(\psi)}\right) ;
$$

(ii) there exists $C$ depending only on $\lambda, \Lambda, \kappa,\left\|a_{i j}\right\|_{0, \alpha, \Omega_{e}},\left\|b_{i}\right\|_{1, \alpha, \Omega_{e}}$, and $\Omega$ such that

$$
\|u\|_{2, \alpha, \Omega^{+}(\psi)} \leq C\left(\|r\|_{0, \alpha, \Omega^{+}(\psi)}+\|g\|_{1, \alpha, \Omega^{+}(\psi)}\right) .
$$

We now prove this lemma. Consider the functions

$$
\underline{u}(x):=K\left(e^{x_{n}}-e^{N_{2}}\right), \quad \bar{u}(x):=-K\left(e^{x_{n}}-e^{N_{2}}\right),
$$

with $K=C_{1}\left(\|g\|_{0, \Omega^{+}(\psi)}+\|r\|_{0, \Omega^{+}(\psi)}\right)$, where $C_{1}$ will be chosen below depending only on the data as in (ii). Then, using (4.4) and the ellipticity of $\overline{\mathcal{L}}$, we get

$$
\overline{\mathcal{L}}[\underline{u}]=a_{n n} K e^{x_{n}} \geq C_{1} \lambda e^{-C M \sigma}\|r\|_{0, \Omega^{+}(\psi)} \geq\|r\|_{0, \Omega^{+}(\psi)},
$$


if $M \sigma \leq 1$ and $C_{1} \geq e^{C}$. Similarly, for such $\sigma$ and $C_{1}$,

$$
\overline{\mathcal{L}}[\bar{u}] \leq-\|r\|_{0, \Omega^{+}(\psi)} .
$$

In addition,

$$
\underline{u}=\bar{u}=0 \quad \text { on } \Gamma_{1} .
$$

Let $w:=\underline{u}-u$. Then

$$
\overline{\mathcal{L}} w \geq\|r\|_{0, \Omega^{+}(\psi)}-r \geq 0 \quad \text { in } \Omega^{+}(\psi),
$$

i.e., $w$ is a subsolution of $\overline{\mathcal{L}}$. Thus, by the strong maximum principle, if the maximum of $w$ is achieved in the interior of $\Omega^{+}(\psi)$, then $w=$ const. in $\Omega^{+}(\psi)$, and thus $w \equiv 0$ in $\Omega^{+}(\psi)$ since $\left.w\right|_{\Gamma_{1}}=0$.

Let the maximum of $w$ be attained at $x_{0} \in S_{\psi}$. Then $w_{\tau}\left(x_{0}\right)=0$ and thus, since $b\left(x_{0}\right) \cdot \nu\left(x_{0}\right)>0$, we get

$$
w_{\nu}\left(x_{0}\right)=\frac{1}{b\left(x_{0}\right) \cdot \nu\left(x_{0}\right)} b\left(x_{0}\right) \cdot D w\left(x_{0}\right)=\frac{1}{b\left(x_{0}\right) \cdot \nu\left(x_{0}\right)}\left(b\left(x_{0}\right) \cdot D \underline{u}\left(x_{0}\right)-g\left(x_{0}\right)\right) .
$$

Using (4.8) and choosing $\sigma$ small, depending only on $n, \gamma, q^{+}, \Omega$, and $M$, and choosing $C_{1}$ sufficiently large, depending only upon the data as in (ii), we obtain, on $S_{\psi}$,

$$
\begin{aligned}
b \cdot D \underline{u} & =K e^{x_{n}} b \cdot \nu_{0} \geq K e^{x_{n}}\left(b \cdot \nu+b \cdot\left(\nu_{0}-\nu\right)\right) \\
& \geq C_{1} e^{-C M \sigma}(\kappa-\Lambda C M \sigma)\|g\|_{0, \Omega^{+}(\psi)} \geq\|g\|_{0, \Omega^{+}(\psi)} .
\end{aligned}
$$

Then

$$
w_{\nu}\left(x_{0}\right) \geq \frac{1}{b\left(x_{0}\right) \cdot \nu\left(x_{0}\right)}\left(\|g\|_{0, \Omega^{+}(\psi)}-g\left(x_{0}\right)\right) \geq 0,
$$

which contradicts the Hopf Lemma. Thus,

$$
\sup _{\Omega^{+}(\psi)}(\underline{u}-u)=\sup _{\Gamma_{1}}(\underline{u}-u)=0 .
$$

This and similar argument for $\bar{u}$ imply

$$
\underline{u}(x) \leq u(x) \leq \bar{u}(x), \quad \text { for any } x \in \Omega^{+}(\psi),
$$

which implies (4.22). This also implies the uniqueness of classical solutions of (4.21).

Furthermore, let

$$
\Omega_{0}^{+}:=\mathbf{T}^{n-1} \times(0,1) .
$$

Define a flattening map $\Phi: \Omega^{+}(\psi) \rightarrow \Omega_{0}^{+}$, which maps $S_{\psi}$ to $\left\{x_{n}=0\right\}$, by

$$
\Phi\left(x^{\prime}, x_{n}\right)=\left(x^{\prime}, \frac{x_{n}-f\left(x^{\prime}\right)}{N_{2}-f\left(x^{\prime}\right)}\right)
$$

for the function $f\left(x^{\prime}\right)$ in (4.4). Note that, from the estimate for $f\left(x^{\prime}\right)$ in (4.4),

$$
\|\Phi-I d\|_{2, \alpha, \Omega^{+}(\psi)},\left\|\Phi^{-1}-I d\right\|_{2, \alpha, \Omega_{0}^{+}} \leq \frac{1}{10},
$$

if $\sigma$ is small. Now, consider $u(x)$ as a periodic solution with respect to the $x^{\prime}$ variables in $\mathbf{R}^{n-1} \times\left[-N_{1}, N_{2}\right] \cap\left\{x_{n}>f\left(x^{\prime}\right)\right\}$. Then, (4.23) follows from the Schauder estimates (see Theorem 6.2, Corollary 6.7, and Lemma 6.29 in [17]) and from (4.22); the fact that $C$ is independent of $M, \sigma$, and $\psi$ follows from (4.26). This completes the proof of Lemma 4.2

From Lemma 4.2, we easily have 
Lemma 4.3. Let $\sigma$ be sufficiently small, depending only on $n, \gamma, q^{+}, \Omega$, and $M$. Then there exists a unique solution $u \in C^{2, \alpha}\left(\overline{\Omega^{+}(\psi)}\right)$ of (4.21) satisfying (4.23) with $C$ depending only on $\lambda, \Lambda, \kappa,\left\|a_{i j}\right\|_{0, \alpha, \Omega_{e}}$, and $\left\|b_{i}\right\|_{1, \alpha, \Omega_{e}}$.

The proof is based on some standard existence argument for elliptic equations; we sketch it in Appendix A.

Step 3. Now we turn to the nonlinear problem (4.14)-(4.16). Rewrite equation (4.14) in the nondivergence form:

$$
\mathcal{N}[v]:=\sum_{i, j=1}^{n} A^{i j}(D v) v_{x_{i} x_{j}}=0,
$$

where $A_{i j}(P):=A_{P_{j}}^{i}(P), P \in \mathbf{R}^{n}$. From the definition of $A(P)$, (3.3), and (3.4),

$$
\begin{aligned}
& A_{i j}(P)=A_{j i}(P), \\
& (1+|P|)\left|D_{P} A_{i j}(P)\right| \leq \hat{C},
\end{aligned}
$$

for any $P \in \mathbf{R}^{n}, i, j=1, \cdots, n$.

The unique solvability of the linear problem (4.21) allows us to use the nonlinear method of continuity to solve (4.27), (4.15), and (4.16). Namely, in order to show the existence and uniqueness of a solution $v \in C^{2, \alpha}\left(\overline{\Omega^{+}(\psi)}\right)$ of (4.27), (4.15), and (4.16), by Lemma 17.29 and Theorem 17.7 in [17], it suffices to verify the following estimate.

Lemma 4.4. For any solution $u \in C^{2, \alpha}\left(\overline{\Omega^{+}(\psi)}\right)$ of the problem:

$$
\begin{aligned}
& \sum_{i, j=1}^{n} A_{i j}(D u(x)) u_{x_{i} x_{j}}=0 \quad \text { in } \Omega^{+}(\psi), \\
& \sum_{i=1}^{n} A^{i}(D u) \nu^{i}=t g_{\psi} \quad \text { on } S_{\psi}, \\
& u=0 \quad \text { on } \Gamma_{1},
\end{aligned}
$$

the estimate

$$
\|u\|_{1, \delta, \Omega^{+}(\psi)} \leq C
$$

holds with some $\delta>0$ for any $t \in[0,1]$, where $C$ and $\delta$ depend only on $n, \lambda, \Lambda$, the constant $\hat{C}$ from (4.29), $\|g\|_{1, \alpha, \Omega^{+}(\psi)}, S_{\psi}$, and $\Omega$, but are independent of $t$.

The proof of Lemma 4.4 follows from some well-known estimates for nonlinear elliptic equations of second order; we outline the proof in Appendix B.

Note that the constants $C$ and $\delta$ in (4.33) and (4.34) are independent of $M$ and $\psi \in \mathcal{K}_{M}$, if $M \sigma$ is small. Indeed, the dependence of $C$ and $\delta$ in (4.33) on $S_{\psi}$ is through the estimates of derivatives of the regularized distance function $\rho(x)$ described in [24, page 522]. These are estimated in terms of the $C^{2, \alpha}$-norm of $S_{\psi}$ 24. page 522], which, in our case, is determined by the $C^{2, \alpha}$ norm of $f\left(x^{\prime}\right)$ from (4.4) and estimated by $C M \sigma$. Thus, if $\psi \in \mathcal{K}_{M}$ and $M \sigma \leq 1$, then $C$ and $\delta$ in (4.33) depend only on $n, \gamma, q^{+}, \Omega$, and $\left\|g_{\psi}\right\|_{1, \alpha, \Omega_{e}}$.

Moreover, it follows from [23. Theorem 2] that the solution $v \in C^{2}\left(\overline{\Omega^{+}(\psi)}\right)$ of (4.27), (4.15), and (4.16) satisfies $v \in C^{2, \alpha}\left(\overline{\Omega^{+}(\psi)}\right)$, and

$$
\|v\|_{2, \alpha, \Omega^{+}(\psi)} \leq C
$$


where $C$ depends only on the same quantities as the constants in (4.33), i.e., on $n$, $\gamma, q^{+}, \Omega$, and $\left\|g_{\psi}\right\|_{1, \alpha, \Omega_{e}}$.

By (4.17), the solution $v(x)$ of (4.27), (4.15), and (4.16) satisfies a linear problem of the form (4.21) with

$$
\begin{aligned}
& a_{i j}(x)=A_{P_{j}}^{i}(D v(x)), \\
& b_{i}(x)=\sum_{j=1}^{n} \int_{0}^{1} A_{P_{i}}^{j}(s D v(x)) d s \nu^{j}(x), \\
& r(x) \equiv 0 .
\end{aligned}
$$

Then, using (4.20) and (4.34), we conclude that the functions defined by 4.35) satisfy $\left\|a_{i j}\right\|_{1, \alpha, \Omega^{+}(\psi)}+\left\|b_{i}\right\|_{1, \alpha, \Omega^{+}(\psi)} \leq C$, where $C$ depends only on $n, \gamma, q^{+}$, and $\Omega$ if $M \sigma \leq 1$. Thus, using (4.23) for $v(x)$, with $r(x)=0$ and $g=g_{\psi}$, and recalling (4.20), we obtain

$$
\|v\|_{2, \alpha, \Omega^{+}(\psi)} \leq C \sigma
$$

Thus, $\varphi(x)=v(x)+\varphi_{0}^{+}(x)$ is the unique solution of (4.10)-4.12) and satisfies (4.13).

Step 4. Now we show that $\varphi(x)$ satisfies (3.9). Since $\psi(x)$ satisfies (3.9), it follows that $G_{\psi}(x)$ and $\Omega^{+}(\psi)$, i.e., the function $f(x)$ in (4.4), satisfy (3.9). Fix any $m \in\{1, \cdots, n-1\}$ and $k \in\{0, \pm 1, \pm 2, \cdots\}$, and let

$$
v(x):=\varphi\left(x_{1}, \cdots, x_{m-1}, 2 k a-x_{m}, x_{m+1}, \cdots, x_{n}\right) .
$$

Then $v(x)$ is a solution of (4.10)-4.12): Indeed, since $G_{\psi}(x)$ and $\Omega^{+}(\psi)$ satisfy (3.9), the only fact we should check is that, if $\varphi(x)$ is a solution of (4.10), then $v(x)$ also satisfies (4.10). This follows from the structure of (4.10) and is readily checked by a direct calculation. Thus, by the uniqueness of solutions of the problem (4.10)-(4.12), we obtain $\varphi(x) \equiv v(x)$, and so $\varphi(x)$ satisfies (3.9).

4.3. Construction and continuity of the iteration map. Now we first construct the iteration map by an extension of the unique solution of (4.10)-(4.12) satisfying (4.13). Then we show the continuity of the iteration map.

Proposition 4.5. Let $M \geq 1$. Let $\sigma>0, \varphi^{-}$, and $\psi$ be as those in Proposition 4.1. Let $\varphi$ be a solution of the problem (4.10)-(4.12) in the domain $\Omega^{+}(\psi)$. Then $\varphi$ can be extended to the whole domain $\Omega_{e}$ such that the extension, denoted by $\mathcal{P}_{\psi} \varphi$, satisfies the following two properties:

(i) There exists $C_{0}>0$, which depends only on $n, \gamma, q^{+}$, and $\Omega$, and is independent of $M, \sigma$, and $\psi$, such that

$$
\left\|\mathcal{P}_{\psi} \varphi-\varphi_{0}^{+}\right\|_{2, \alpha, \Omega_{e}} \leq C_{0} \sigma .
$$

(ii) Let $\beta \in(0, \alpha)$. Let a sequence $\psi_{j} \in \mathcal{K}_{M}$ converge in $C^{2, \beta}\left(\overline{\Omega_{e}}\right)$ to $\psi \in$ $\mathcal{K}_{M}$. Let $\varphi_{j} \in C^{2, \alpha}\left(\overline{\Omega^{+}\left(\psi_{j}\right)}\right)$ and $\varphi \in C^{2, \alpha}\left(\overline{\Omega^{+}(\psi)}\right)$ be the solutions of the problems (4.10)-4.12) for $\psi_{j}$ and $\psi$, respectively. Then $\mathcal{P}_{\psi_{j}} \varphi_{j} \rightarrow \mathcal{P}_{\psi} \varphi$ in $C^{2, \beta}\left(\overline{\Omega_{e}}\right)$.

Proof. We divide the proof into four steps.

Step 1. Let

$$
\kappa=\frac{N_{1}}{N_{2}} .
$$


Denote

$$
\Omega_{0}:=\mathbf{T}^{n-1} \times(-2 \kappa, 1), \quad \Omega_{0}^{+}:=\mathbf{T}^{n-1} \times(0,1) .
$$

We first employ the extension map in [17, pp. 136-137] to define an extension operator $\mathcal{E}_{2}: C^{2, \beta}\left(\overline{\Omega_{0}^{+}}\right) \rightarrow C^{2, \beta}\left(\overline{\Omega_{0}}\right)$ for any $\beta \in(0,1)$. Let $v \in C^{2, \beta}\left(\overline{\Omega_{0}^{+}}\right)$. Define $\mathcal{E}_{2} v=v$ in $\Omega_{0}^{+}$. For $\left(x^{\prime}, x_{n}\right) \in \mathbf{T}^{n-1} \times(-2 \kappa, 0)$, define

$$
\mathcal{E}_{2} v\left(x^{\prime}, x_{n}\right)=\sum_{i=1}^{3} c_{i} v\left(x^{\prime},-\frac{x_{n}}{2 \kappa i}\right),
$$

where $c_{1}, c_{2}$, and $c_{3}$ are constants determined by the system of equations

$$
\sum_{i=1}^{3} c_{i}\left(-\frac{1}{2 \kappa i}\right)^{m}=1, \quad m=0,1,2 .
$$

It is easy to see that $\mathcal{E}_{2} v \in C^{2, \beta}\left(\overline{\Omega_{0}}\right)$ and

$$
\left\|\mathcal{E}_{2} v\right\|_{2, \beta, \Omega_{0}} \leq C\|v\|_{2, \beta, \Omega_{0}^{+}},
$$

with a uniform constant $C$. Since $\mathcal{E}_{2}$ is a linear operator, it follows that $\mathcal{E}_{2}$ : $C^{2, \beta}\left(\overline{\Omega_{0}^{+}}\right) \rightarrow C^{2, \beta}\left(\overline{\Omega_{0}}\right)$ is continuous.

The extension map $\mathcal{E}_{2}$ has also the following properties:

(1) If $v$ satisfies (3.9), then so does $\mathcal{E}_{2} v$.

(2) Let $v_{j} \in C^{2, \beta}\left(\overline{\Omega_{0}^{+}}\right)$and $\mathcal{E}_{2} v_{j} \rightarrow v$ in $C^{2, \beta}\left(\overline{\Omega_{0}}\right)$ as $j \rightarrow \infty$. Then

$$
v=\mathcal{E}_{2}\left(\left.v\right|_{\Omega_{0}^{+}}\right) \quad \text { in } \Omega_{0} \text {. }
$$

Assertion (1) follows directly from the definition of $\mathcal{E}_{2}$. Assertion (2) follows from the continuity of $\mathcal{E}_{2}$. Indeed, since $\left.v_{j} \rightarrow v\right|_{\Omega_{0}^{+}}$, then $\mathcal{E}_{2} v_{j} \rightarrow \mathcal{E}_{2}\left(\left.v\right|_{\Omega_{0}^{+}}\right)$, which implies (2).

Step 2. We first point out the following elementary fact, whose proof can be readily obtained by explicit calculations.

Lemma 4.6. Let $\Omega_{1}, \Omega_{2} \subset \mathbf{R}^{n}$ be bounded, open sets. Let $f \in C^{2, \beta}\left(\bar{\Omega}_{2}\right)$ and $\Phi: \Omega_{1} \rightarrow \Omega_{2}$ satisfy $\Phi \in C^{2, \beta}\left(\bar{\Omega}_{1} ; \mathbf{R}^{n}\right)$. Then $f \circ \Phi \in C^{2, \beta}\left(\bar{\Omega}_{1}\right)$ and

$$
\|f \circ \Phi\|_{2, \beta, \Omega_{1}} \leq C\|f\|_{2, \beta, \Omega_{2}}
$$

where $C=C\left(n,\|\Phi\|_{2, \beta, \Omega_{1}}\right)$. Also, if $f_{j} \rightarrow f$ in $C^{2, \beta}\left(\bar{\Omega}_{2}\right)$ and if $\Phi_{j} \rightarrow \Phi$ in $C^{2, \beta}\left(\bar{\Omega}_{1} ; \mathbf{R}^{n}\right)$, then

$$
f_{j} \circ \Phi_{j} \rightarrow f \circ \Phi \quad \text { in } C^{2, \beta}\left(\bar{\Omega}_{1}\right) .
$$

Now let $\psi \in \mathcal{K}_{M}$. We define the extension operator $\mathcal{E}_{\psi}: C^{2, \beta}\left(\overline{\Omega^{+}(\psi)}\right) \rightarrow$ $C^{2, \beta}\left(\overline{\Omega_{e}}\right)$ for any $\beta \in(0, \alpha]$ as follows. Let $\Phi: \Omega^{+}(\psi) \rightarrow \Omega_{0}^{+}$be a map that flattens $S_{\psi}$ and is defined by (4.25), which in fact defines $\Phi: \Omega_{e} \rightarrow \mathbf{T}^{n-1} \times \mathbf{R}$. This map is $C^{2, \alpha}$ and, if $M \sigma$ is small enough, then

$$
\mathbf{T}^{n-1} \times\left[-\frac{\kappa}{2}, 1\right] \subset \Phi\left(\Omega_{e}\right) \subset \mathbf{T}^{n-1} \times\left[-\frac{3 \kappa}{2}, 1\right] .
$$

Also, if $M \sigma$ is small, then the inverse $\Phi^{-1}: \Phi\left(\Omega_{e}\right) \rightarrow \Omega_{e}$ exists and, similar to (4.26),

$$
\|\Phi-I d\|_{2, \alpha, \Omega_{e}},\left\|\Phi^{-1}-I d\right\|_{2, \alpha, \Phi\left(\Omega_{e}\right)} \leq \frac{1}{10}
$$


Then, for $v \in C^{2, \beta}\left(\overline{\Omega^{+}(\psi)}\right)$, we define

$$
\mathcal{E}_{\psi} v=\left[\mathcal{E}_{2}\left(v \circ \Phi^{-1}\right)\right] \circ \Phi .
$$

This is well defined by the right inclusion in (4.38). From (4.37), (4.39), and Lemma 4.6, we obtain that, if $\sigma$ is so small that (4.38) and (4.39) hold, then, for any $\psi \in \mathcal{K}_{M}$ and $v \in C^{2, \beta}\left(\overline{\Omega_{e}}\right)$,

$$
\left\|\mathcal{E}_{\psi} v\right\|_{2, \beta, \Omega_{e}} \leq C\|v\|_{2, \beta, \Omega^{+}(\psi)}
$$

with $C$ depending only on $n$ and $\Omega$. Since the map $\mathcal{E}_{\psi}: C^{2, \beta}\left(\overline{\Omega^{+}(\psi)}\right) \rightarrow C^{2, \beta}\left(\overline{\Omega_{e}}\right)$ is linear, it follows that this map is continuous.

Step 3. The map $\mathcal{E}_{\psi}$ satisfies the following properties.

Lemma 4.7. Assume that $M \sigma$ is small and $\beta \in(0, \alpha]$.

(i) If $\psi \in \mathcal{K}_{M}$ and $v \in C^{2, \beta}\left(\overline{\Omega^{+}(\psi)}\right)$ satisfies (3.9), then $\mathcal{E}_{\psi} v$ also satisfies (3.9).

(ii) Let $\psi_{j} \in \mathcal{K}_{M}$ and $\psi_{j} \rightarrow \psi$ in $C^{2, \beta}\left(\overline{\Omega_{e}}\right)$ as $j \rightarrow \infty$. Let $v_{j} \in C^{2, \beta}\left(\overline{\Omega^{+}\left(\psi_{j}\right)}\right)$ and $\mathcal{E}_{\psi_{j}} v_{j} \rightarrow v$ in $C^{2, \beta}\left(\overline{\Omega_{e}}\right)$ as $j \rightarrow \infty$. Then

$$
v=\mathcal{E}_{\psi}\left(\left.v\right|_{\Omega^{+}(\psi)}\right) \quad \text { in } \Omega_{e} .
$$

These can be seen as follows. Assertion (ii) follows from the definition of $\mathcal{E}_{\psi}$ and the property (i) of $\mathcal{E}_{2}$ in Step 1, since the maps $\Phi$ and $\Phi^{-1}$ satisfy (3.9), which follows from (4.25) because $\psi$ satisfies 3.9.

Now we prove (iii). Since $\psi_{j} \in \mathcal{K}_{M}$ and $\psi_{j} \rightarrow \psi$ in $C^{2, \beta}$, then $\psi \in \mathcal{K}_{M}$. Let $f_{j}$ and $f$ be the functions from (4.4) for $\psi_{j}$ and $\psi$, respectively. Since $\psi_{j} \rightarrow \psi$ in $C^{2, \beta}$ and (4.3) holds, then

$$
f_{j} \rightarrow f \quad \text { in } C^{2, \beta}\left(\mathbf{T}^{n-1}\right) .
$$

Let $\Phi_{j}: \Omega_{e} \rightarrow \mathbf{T}^{n-1} \times \mathbf{R}$ be the flattening map (4.25) corresponding to $\psi_{j}$. It follows that

$$
\Phi_{j} \rightarrow \Phi \quad \text { in } C^{2, \beta}\left(\Omega_{e} ; \mathbf{T}^{n-1} \times \mathbf{R}\right) .
$$

Also, by (4.38), it follows that

$$
\Phi_{j}^{-1} \rightarrow \Phi^{-1} \quad \text { in } C^{2, \beta}\left(\mathbf{T}^{n-1} \times\left[-\frac{\kappa}{2}, 1\right] ; \Omega_{e}\right) .
$$

Let $w \in C^{2, \beta}\left(\overline{\Phi\left(\Omega_{e}\right)}\right)$ and $w_{j} \in C^{2, \beta}\left(\overline{\Omega_{0}^{+}}\right)$be defined by $w=v \circ \Phi^{-1}$ and $w_{j}=$ $v_{j} \circ \Phi_{j}^{-1}$, which is well defined since $\Phi_{j}\left(\Omega^{+}\left(\psi_{j}\right)\right)=\Omega_{0}^{+}$. Then, using the first inclusion in (4.38) and the second assertion of Lemma 4.6 we have

$$
w_{j} \rightarrow w \quad \text { in } C^{2, \beta}\left(\overline{\Omega_{0}^{+}}\right) .
$$

Thus, using the continuity of $\mathcal{E}_{2}$, we have

$$
\mathcal{E}_{2} w_{j} \rightarrow \mathcal{E}_{2}\left(\left.w\right|_{\Omega_{0}^{+}}\right) \quad \text { in } C^{2, \beta}\left(\overline{\Omega_{0}}\right) .
$$

Using the second inclusion in (4.38) for $\Phi$ and $\Phi_{j}$, we get

$$
\left(\mathcal{E}_{2} w_{j}\right) \circ \Phi_{j} \rightarrow \mathcal{E}_{2}\left(\left.w\right|_{\Omega_{0}^{+}}\right) \circ \Phi \quad \text { in } C^{2, \beta}\left(\overline{\Omega_{e}}\right),
$$

which is

This implies (ii).

$$
\mathcal{E}_{\psi_{j}} v_{j} \rightarrow \mathcal{E}_{\psi}\left(\left.v\right|_{\Omega^{+}(\psi)}\right) \quad \text { in } C^{2, \beta}\left(\overline{\Omega_{e}}\right)
$$


Step 4. Finally, we define the following extension operator $\mathcal{P}_{\psi}: C^{2, \beta}\left(\overline{\Omega^{+}(\psi)}\right) \rightarrow$ $C^{2, \beta}\left(\overline{\Omega_{e}}\right)$ for $\psi \in \mathcal{K}_{M}$ and $\beta \in(0, \alpha]$ :

$$
\mathcal{P}_{\psi} v=\mathcal{E}_{\psi}\left(v-\varphi_{0}^{+}\right)+\varphi_{0}^{+}, \quad \text { where } v \in C^{2, \alpha}\left(\overline{\Omega^{+}(\psi)}\right) .
$$

The estimate (4.36) follows from (4.13), (4.41), and (4.43).

Now we prove the assertion (iii) of Proposition 4.5. Let $0<\beta<\alpha$. By (4.36),

$$
\left\|\mathcal{P}_{\psi_{j}} \varphi_{j}\right\|_{2, \alpha, \Omega_{e}} \leq C .
$$

Thus, there exists a subsequence (still denoted) $\varphi_{j}$ such that

$$
\mathcal{P}_{\psi_{j}} \varphi_{j} \rightarrow v \quad \text { in } C^{2, \beta}\left(\overline{\Omega_{e}}\right),
$$

for some $v \in C^{2, \alpha}\left(\overline{\Omega_{e}}\right)$.

Denoting $f_{j}$ as the function from (4.4) for $\psi_{j}$ as above and using (4.42) yield that $v$ satisfies equation (4.10) in $\Omega^{+}(\psi)$. Also, $v$ obviously satisfies condition (4.12). Now we show that $v(x)$ also satisfies condition (4.11) on $S_{\psi}$. Denote $\nu_{j}$ as the function (4.7) corresponding to $\psi_{j}$. Then

$$
\nu_{j} \rightarrow \nu \quad \text { in } C^{1, \beta}\left(\Omega_{e}\right) .
$$

Let $x^{\prime} \in \mathbf{T}^{n-1}$. Denote $x_{j}=\left(x^{\prime}, f_{j}\left(x^{\prime}\right)\right)$ and $x=\left(x^{\prime}, f\left(x^{\prime}\right)\right)$. By (4.42), $x_{j} \rightarrow x$. Since $\varphi_{j}$ satisfies (4.11) on $S_{\psi_{j}}$, we have

$$
\tilde{\rho}\left(\left|D \varphi_{j}\left(x_{j}\right)\right|^{2}\right) D \varphi_{j}\left(x_{j}\right) \cdot \nu_{j}\left(x_{j}\right)=G_{\psi_{j}}\left(x_{j}\right) .
$$

By (4.9) and 4.45),

$$
G_{\psi_{j}} \rightarrow G_{\psi} \quad \text { in } C^{1, \beta}\left(\Omega_{e}\right)
$$

Then we have

$$
\begin{aligned}
\tilde{\rho}\left(|D \varphi(x)|^{2}\right) D v(x) \cdot \nu(x) & =\lim _{j \rightarrow \infty} \tilde{\rho}\left(\left|D \varphi_{j}\left(x_{j}\right)\right|^{2}\right) D \varphi_{j}\left(x_{j}\right) \cdot \nu_{j}\left(x_{j}\right) \\
& =\lim _{j \rightarrow \infty} G_{\psi_{j}}\left(x_{j}\right) \\
& =G_{\psi}(x) .
\end{aligned}
$$

Since $S_{\psi}=\left\{\left(x^{\prime}, f\left(x^{\prime}\right)\right): x^{\prime} \in \mathbf{T}^{n-1}\right\}$, we conclude that $v$ satisfies (4.11). 4.1 ,

Thus, $v$ is a solution of (4.10)-(4.12) in $\Omega^{+}(\psi)$. By the uniqueness in Proposition

$$
\left.v\right|_{\Omega^{+}(\psi)}=\varphi .
$$

Then, by (4.43), (4.44), and Lemma 4.7(1ii), we have

$$
v=\mathcal{E}_{\psi}\left(\left.\left(v-\varphi_{0}^{+}\right)\right|_{\Omega^{+}(\psi)}\right)+\varphi_{0}^{+}=\mathcal{E}_{\psi}\left(\varphi-\varphi_{0}^{+}\right)+\varphi_{0}^{+}=\mathcal{P}_{\psi} \varphi \quad \text { in } \quad \Omega_{e} .
$$

We have thus proved that a subsequence of $\mathcal{P}_{\psi_{j}} \varphi_{j}$ converges to $\mathcal{P}_{\psi} \varphi$ in $C^{2, \beta}\left(\overline{\Omega_{e}}\right)$. Moreover, by the same argument, from any subsequence of $\mathcal{P}_{\psi_{j}} \varphi_{j}$ we can extract a further subsequence, converging in $C^{2, \beta}\left(\overline{\Omega_{e}}\right)$ to the same limit $\mathcal{P}_{\psi} \varphi$. Thus the whole sequence $\mathcal{P}_{\psi_{j}} \varphi_{j}$ converges to $\mathcal{P}_{\psi} \varphi$. Proposition 4.5 is proved. 
4.4. Existence of solutions of the free boundary problem. With Sections 4.1 4.3. we can now prove the existence of solutions of the free boundary problem.

Define the iteration map $J: \mathcal{K}_{M} \rightarrow C^{2, \alpha}\left(\overline{\Omega_{e}}\right)$ by

$$
J \psi:=\mathcal{P}_{\psi} \varphi
$$

where $\varphi$ is the unique solution of the problem (4.10)-(4.12) for $\psi$. By Proposition 4.5.(ii), $J$ is continuous in the $C^{2, \beta}\left(\overline{\Omega_{e}}\right)$-norm for any positive $\beta<\alpha$.

Now we denote by $\varphi$ both the function $\varphi$ in $\Omega^{+}(\psi)$ and its extension $\mathcal{P}_{\psi} \varphi$.

Choose $M$ to be the constant $C_{0}$ from (4.36). Then, for $\psi \in \mathcal{K}_{M}$, we have from Proposition 4.5(1i) that $\varphi:=J \psi \in \mathcal{K}_{M}$ if $\sigma>0$ is sufficiently small depending only on $n, \gamma, q^{+}$, and $\Omega$, since $M$ is now fixed. Thus, (4.47) defines the iteration map $J: \mathcal{K}_{M} \rightarrow \mathcal{K}_{M}$ and, from Proposition 4.5(iii), $J$ is continuous on $\mathcal{K}_{M}$ in the $C^{2, \beta}\left(\overline{\Omega_{e}}\right)$-norm for any positive $\beta<\alpha$.

In order to find a classical solution of Problem $\mathrm{C}$, we seek a fixed point of the map J. We use the Schauder Fixed Point Theorem (cf. [17, Theorem 11.1]) in the following setting:

Let $\sigma>0$ satisfy the conditions of Proposition 4.5. Let $\beta \in(0, \alpha)$. Since $\Omega_{e}$ is a compact manifold with boundary, the set $\mathcal{K}_{M}$ is a compact convex subset of $C^{2, \beta}\left(\overline{\Omega_{e}}\right)$. We have shown that $J\left(\mathcal{K}_{M}\right) \subset \mathcal{K}_{M}$, and $J$ is continuous in the $C^{2, \beta}\left(\overline{\Omega_{e}}\right)$ norm. Then, by the Schauder Fixed Point Theorem, $J$ has a fixed point $\varphi \in \mathcal{K}_{M}$.

If $\varphi$ is such a fixed point, then

$$
\tilde{\varphi}(x):=\min \left(\varphi^{-}(x), \varphi(x)\right)
$$

is a classical solution of Problem $\mathrm{C}$, and $S_{\varphi}$ is its free boundary.

It follows that $\tilde{\varphi}$ is a solution of Problem $\mathrm{B}$, provided that $\sigma$ is small enough so that (4.13) implies that $|D \varphi|<p_{\text {sonic }}^{1}-\varepsilon$, where $\varepsilon$ is defined by (3.2). Indeed, then (3.5) implies that $\varphi$ lies in the nontruncated region for the equation (3.6).

For such values of $\sigma$, the function $\tilde{\varphi}$ is a solution of Problem A. Indeed, $|D \tilde{\varphi}|<$ $p_{\text {sonic }}^{1}-\varepsilon$ on $\Omega^{+}(\tilde{\varphi}):=\left\{\tilde{\varphi}<\varphi^{-}\right\}$since $\tilde{\varphi}=\varphi$ on $\Omega^{+}(\tilde{\varphi})$.

This completes the existence proof for Theorems 2.1 and 2.2.

\section{UNIQUENESS OF SOLUTIONS}

In this section, we prove the uniqueness of solutions of Problem B (hence, Problem A) that we have constructed in Section 4.

Theorem 5.1. Let $q^{+}, q^{-}$, and $\varphi^{-}$be as in Theorem 2.1. Let $M>0$. If $\sigma>0$ is a sufficiently small constant depending only on $M, n, q^{+}, \gamma$, and $\Omega$, then there exists at most one solution $\varphi$ of Problem $B$ satisfying

$$
\left\|\varphi-\varphi_{0}^{+}\right\|_{2, \alpha, \Omega^{+}(\varphi)} \leq M \sigma .
$$

Proof. In this proof, the constants $\sigma, C$, and $c$ depend only on $M, n, q^{+}, \gamma$, and $\Omega$, unless other dependence is specified. The proof consists of seven steps.

Step 1. We consider Problem B extended to the domain $\Omega_{e}$. Let $\varphi \neq \hat{\varphi}$ be two solutions of Problem B satisfying (5.1). Define

$$
u:=\varphi^{-}-\varphi, \quad \hat{u}:=\varphi^{-}-\hat{\varphi} \quad \text { in } \Omega_{e} .
$$

Then

$$
u \geq 0, \quad \hat{u} \geq 0 \quad \text { in } \Omega_{e},
$$

and

$$
\Omega^{+}(\varphi)=\{u(x)>0\} \cap \Omega_{e}, \quad \Omega^{+}(\hat{\varphi})=\{\hat{u}(x)>0\} \cap \Omega_{e} .
$$


Below, $\Omega^{+}(u), S(u), \Omega^{+}(\hat{u})$, and $S(\hat{u})$ stand for $\Omega^{+}(\varphi), S(\varphi), \Omega^{+}(\hat{\varphi})$, and $S(\hat{\varphi})$, respectively. Note that

$$
S(u)=\partial\{u(x)>0\} \cap \Omega_{e}, \quad S(\hat{u})=\partial\{\hat{u}(x)>0\} \cap \Omega_{e} .
$$

The definition of $u$ and $\hat{u}$ with (5.1) implies

$$
\left\|u-\left(q^{-}-q^{+}\right) x_{n}\right\|_{2, \alpha, \Omega^{+}(u)} \leq M \sigma, \quad\left\|\hat{u}-\left(q^{-}-q^{+}\right) x_{n}\right\|_{2, \alpha, \Omega^{+}(\hat{u})} \leq M \sigma .
$$

If (4.2) holds with large enough $C$, then the regions $\Omega^{+}(u)$ and $\Omega^{+}(\hat{u})$ have the form (4.4) with the functions $f$ and $\hat{f}$, respectively. To see this, we use (5.2) and extend $u$ from $\Omega^{+}(u)$, which we consider now as a subset of $\mathbf{R}^{n}$, into $\mathbf{R}^{n}$ so that the extension $\mathcal{E} u$ satisfies

$$
\left\|\mathcal{E} u-\left(q^{-}-q^{+}\right) x_{n}\right\|_{2, \alpha, \mathbf{R}^{n}} \leq C M \sigma,
$$

where $C$ depends only on $n$ (see, e.g., 31, Chapter 6 , Theorem 4]). Then, by (4.2),

$$
(\mathcal{E} u)_{x_{n}}(x) \geq q^{-}-q^{+}-C M \sigma \geq \frac{q^{-}-q^{+}}{2} \geq 0 .
$$

Thus, $\Omega^{+}(u)=\{\mathcal{E} u(x)>0\} \cap \Omega_{e}$, and (4.4) holds for $u$ by the implicit function theorem; and the corresponding results hold for $\hat{u}$.

Step 2. Rewrite the problem (4.10)-(4.12) in terms of $u=\varphi^{-}-\varphi$. It follows from (3.5) and (5.2) that, if $\sigma>0$ is sufficiently small, then $u$ in $\Omega^{+}(u)$ is the solution of the following problem:

$$
\begin{aligned}
& \operatorname{div} A(x, D u)=0 \quad \text { in } \Omega^{+}(u), \\
& u_{\nu}=G_{f} \quad \text { on } S(u), \\
& u=\varphi^{-}-q^{+} N_{2} \quad \text { on } \Gamma_{1}:=\mathbf{T}^{n-1} \times\left\{N_{2}\right\}
\end{aligned}
$$

where

$$
A(y, P)=\tilde{\rho}\left(\left|D \varphi^{-}(y)-P\right|^{2}\right)\left(D \varphi^{-}(y)-P\right) \quad \text { for } y \in \Omega_{e}, \quad P \in \mathbf{R}^{n},
$$

with $\tilde{\rho}$ defined by (3.4).

The function $G_{f}\left(x^{\prime}\right)$ is defined as follows. Using (2.2), we can rewrite (2.5) as

$$
\rho\left(\left|\varphi_{\tau}^{-}\right|^{2}+\left(\varphi_{\nu}^{+}\right)^{2}\right) \varphi_{\nu}^{+}=\rho\left(\left|D \varphi^{-}\right|^{2}\right) \varphi_{\nu}^{-} \quad \text { on } S(u) .
$$

We intend to solve (5.8) for $\varphi_{\nu}^{+}$. Note that, by (2.13),

$$
\left|D \varphi^{-}-q^{-} \nu_{0}\right| \leq \sigma \quad \text { in } \Omega,
$$

and, by (5.3), the unit normal vector $\nu(x)$ to $S(u)$ satisfies

$$
\left|\nu(x)-\nu_{0}\right| \leq C \sigma \text {. }
$$

Thus, noting that

$$
\varphi_{\nu}^{-}=D \varphi^{-} \cdot \nu, \quad\left|\varphi_{\tau}^{-}\right|^{2}=\left|D \varphi^{-}\right|^{2}-\left(\varphi_{\nu}^{-}\right)^{2}=\left|D \varphi^{-}\right|^{2}-\left(D \varphi^{-} \cdot \nu\right)^{2},
$$

we get

Denoting

$$
\left|\varphi_{\nu}^{-}-q^{-}\right| \leq C \sigma, \quad\left|\varphi_{\tau}\right|^{2} \leq C \sigma
$$

$$
K:=1-\theta\left|\varphi_{\tau}^{-}\right|^{2},
$$

we rewrite (5.8) as the equation:

$$
\Phi_{K}(p)=\Phi_{K}\left(D \varphi^{-} \cdot \nu\right),
$$


where $\Phi_{K}(\cdot)$ is the function in Lemma 2.1. Now we solve (5.12) for $p$. For this purpose, based on (5.10) and (5.11), we define

$$
e_{\nu}=e \cdot \nu, \quad\left|e_{\tau}\right|^{2}=|e|^{2}-\left(e_{\nu}\right)^{2}=|e|^{2}-(e \cdot \nu)^{2},
$$

and

$$
K(s, t):=1-\theta\left(s-t^{2}\right), \quad s, t \in \mathbf{R} .
$$

Then

$$
K\left(|e|^{2}, e \cdot \nu\right)=1-\theta\left(|e|^{2}-(e \cdot \nu)^{2}\right)=1-\theta\left|e_{\tau}\right|^{2} \quad \text { for } e \in \mathbf{R}^{n}, \nu \in \mathbf{S}^{n-1} .
$$

Now, (5.12) can be written as

$$
F\left(p ;\left|D \varphi^{-}\right|^{2}, D \varphi^{-} \cdot \nu\right)=0,
$$

where $F \in C^{\infty}$ in the neighborhood of $\left(q^{+} ;\left(q^{-}\right)^{2}, q^{-}\right)$under consideration is defined by

$$
F(p ; s, t):=\Phi_{K(s, t)}(p)-\Phi_{K(s, t)}(t), \quad p, s, t \in \mathbf{R} .
$$

Moreover, by the definition of $q^{+}$and $q^{-}$and Lemma 2.1(iii), we have

$$
F\left(q^{+} ;\left(q^{-}\right)^{2}, q^{-}\right)=0, \quad F_{p}\left(q^{+} ;\left(q^{-}\right)^{2}, q^{-}\right)=\Phi_{1}^{\prime}\left(q^{+}\right)>0 .
$$

Thus, by the Implicit Function Theorem, there exists a $C^{\infty}$ function $G(s, t)$ defined on a neighborhood:

$$
\mathcal{O}_{r_{0}}\left(q^{-}, q^{-}\right)=\left\{(s, t):\left|s-\left(q^{-}\right)^{2}\right|<\delta_{0},\left|t-q^{-}\right|<\delta_{0}\right\},
$$

where $\delta_{0}$ depends only on $q^{+}, q^{-}$, and $\gamma$ such that

$$
G\left(\left(q^{-}\right)^{2}, q^{-}\right)=q^{+},
$$

and

$$
F(G(s, t) ; s, t)=0 .
$$

This means that (5.8) can be rewritten as

$$
\varphi_{\nu}^{+}=G\left(\left|D \varphi^{-}\right|^{2}, D \varphi^{-} \cdot \nu\right),
$$

provided that, on $S(u)$,

$$
\left|D \varphi^{-} \cdot \nu-q^{-}\right|<\delta_{0},\left.\quad|| D \varphi^{-}\right|^{2}-\left(q^{-}\right)^{2} \mid<\delta_{0},
$$

which can be achieved by choosing $\sigma$ small depending only on $n, \gamma, q^{+}, \Omega$, and $M$.

Thus, for $u=\varphi^{-}-\varphi$, (5.5) holds with

$$
G_{f}\left(x^{\prime}\right):=D \varphi^{-}(x) \cdot \nu_{f}(x)-G\left(\left|D \varphi^{-}(x)\right|^{2}, D \varphi^{-}(x) \cdot \nu_{f}(x)\right),
$$

for $x^{\prime} \in \mathbf{T}^{n-1}, x=\left(x^{\prime}, f\left(x^{\prime}\right)\right) \in S(u)$, and $\nu_{f}(x)$ is the inward unit normal vector to $S(u)$ at $x$.

Note that (5.4) is uniformly elliptic with the same ellipticity constants as those for (3.6).

The function $\hat{u}$ is a solution of the similar problem in $\Omega^{+}(\hat{u})$, i.e., $\hat{u}$ satisfies equation (5.4) in $\Omega^{+}(\hat{u})$, (5.6) on $\Gamma_{1}$, and

$$
\hat{u}_{\nu}=G_{\hat{f}} \quad \text { on } S(\hat{u})
$$

where

$$
G_{\hat{f}}\left(x^{\prime}\right)=D \varphi^{-}(x) \cdot \nu_{\hat{f}}(x)-G\left(\left|D \varphi^{-}(x)\right|^{2}, D \varphi^{-}(x) \cdot \nu_{\hat{f}}(x)\right),
$$

for $x^{\prime} \in \mathbf{T}^{n-1}, x=\left(x^{\prime}, \hat{f}\left(x^{\prime}\right)\right) \in S(\hat{u})$, and the inward unit normal vector $\nu_{\hat{f}}(x)$ to $S(\hat{u})$ at $x$. 
Step 3. We may assume $f \neq \hat{f}$; otherwise $\varphi=\hat{\varphi}$ by the uniqueness of solutions to the problem (4.10) - (4.12) in the domain $\Omega^{+}(\varphi)=\Omega^{+}(u)$ by Proposition 4.1 Thus we may assume that $\hat{f}\left(\hat{x^{\prime}}\right)>f\left(\hat{x}^{\prime}\right)$ for some $\hat{x}^{\prime} \in \mathbf{R}^{n-1}$, since the opposite inequality can be handled similarly.

We shift the domain $\Omega^{+}(\hat{u})$ in the direction $-\nu_{0}$ by a distance $\delta>0$ so that the resulting domain $\mathcal{B}$ contains $\Omega^{+}(u) \cap\left\{x_{n}<N_{2}-\delta\right\}$ and $\partial \mathcal{B} \cap S(u) \neq \emptyset$. Precisely, for positive $y<N_{2}$, define $v_{y}: \Omega_{e} \cap\left\{x_{n}<N_{2}-y\right\} \rightarrow \mathbf{R}$ by

$$
v_{y}\left(x^{\prime}, x_{n}\right)=\hat{u}\left(x^{\prime}, x_{n}+y\right) \text {. }
$$

Let

$$
\delta=\sup \left\{y \geq 0: S(u) \cap S\left(v_{y}\right) \neq \emptyset\right\} .
$$

By the above assumption, $\delta>0$. Applying (4.4) to both $f\left(x^{\prime}\right)$ and $\hat{f}\left(x^{\prime}\right)$, we have $\delta \leq C M \sigma$. We assume $C M \sigma<N_{2} / 10$.

We denote $v_{\delta}(x)$ by $v(x)$ and denote $h\left(x^{\prime}\right):=\hat{f}\left(x^{\prime}\right)-\delta$. Clearly,

$$
\Omega^{+}(v)=\left\{\left(x^{\prime}, x_{n}\right): h\left(x^{\prime}\right)<x_{n}<N_{2}-\delta\right\} \cap \Omega_{e} .
$$

It follows that $\Omega^{+}(u) \cap\left\{x_{n}<N_{2}-\delta\right\} \subset \Omega^{+}(v)$. By construction, $f\left(x^{\prime}\right) \geq h\left(x^{\prime}\right)$, and there exists $x_{*}^{\prime} \in \mathbf{T}^{n-1}$ such that $f\left(x_{*}^{\prime}\right)=h\left(x_{*}^{\prime}\right)$. Denote $x_{*}:=\left(x_{*}^{\prime}, f\left(x_{*}^{\prime}\right)\right) \in$ $\mathbf{T}^{n-1} \times \mathbf{R}$. Then the smooth surface $S(u)$ touches the smooth surface $S(v)$ at $x_{*}$. Denote the common unit normal vector to $S(u)$ and $S(v)$ at $x_{*}$ in the direction of $\Omega^{+}(v)$ by $\nu\left(x_{*}\right)$. Since $S(\hat{u})=S(v)+\delta \nu_{0}$, it follows that the inward unit normal vector $\nu_{\hat{f}}\left(x_{*}+\delta \nu_{0}\right)$ to $S(\hat{u})$ at $x_{*}+\delta \nu_{0}=\left(x^{\prime}, f\left(x^{\prime}\right)+\delta\right)$ is equal to $\nu\left(x_{*}\right)$. Then, from the definition of $G_{f}$ and $G_{\hat{f}}$,

$$
\left|G_{f}\left(x_{*}^{\prime}\right)-G_{\hat{f}}\left(x_{*}^{\prime}\right)\right| \leq C\left|D \varphi^{-}\left(x_{*}^{\prime}, f\left(x_{*}^{\prime}\right)\right)-D \varphi^{-}\left(x_{*}^{\prime}, f\left(x_{*}^{\prime}\right)+\delta\right)\right| \leq C \delta \sigma,
$$

where we used (2.13) in the last inequality. Also, since $\hat{u}(x)$ satisfies the free boundary condition $\hat{u}_{\nu}\left(x^{\prime}, \hat{f}\left(x^{\prime}\right)\right)=G_{\hat{f}}\left(x^{\prime}\right)$ and $v(x)=\hat{u}\left(x+\delta \nu_{0}\right)$ for any $x$, we have

$$
v_{\nu}\left(x_{*}\right):=D v\left(x_{*}\right) \cdot \nu\left(x_{*}\right)=D \hat{u}\left(x_{*}+\delta \nu_{0}\right) \cdot \nu_{\hat{f}}\left(x_{*}+\delta \nu_{0}\right)=G_{\hat{f}}\left(x_{*}^{\prime}\right) .
$$

Since $u(x)$ satisfies $u_{\nu}\left(x_{*}\right):=D u\left(x_{*}\right) \cdot \nu\left(x_{*}\right)=G_{f}\left(x_{*}^{\prime}\right)$, we have

$$
\left|v_{\nu}\left(x_{*}\right)-u_{\nu}\left(x_{*}\right)\right|=\left|G_{f}\left(x_{*}^{\prime}\right)-G_{\hat{f}}\left(x_{*}^{\prime}\right)\right| \leq C \sigma \delta .
$$

We will come to a contradiction for small $\sigma$ by showing that $v_{\nu}\left(x_{*}\right)-u_{\nu}\left(x_{*}\right) \geq c \delta$ with $c>0$.

Step 4. Denote $\mathcal{D}:=\Omega^{+}(u) \cap\left\{x_{n}<N_{2}-\delta\right\}$. Then

$$
\partial \mathcal{D}=S(u) \cup\left\{x_{n}=N_{2}-\delta\right\}=\left\{x_{n}=f\left(x^{\prime}\right)\right\} \cup\left\{x_{n}=N_{2}-\delta\right\} .
$$

Then $x_{*} \in \partial \mathcal{D}$, and $\nu\left(x_{*}\right)$ is the inward normal vector to $\partial \mathcal{D}$ at $x_{*}$. Also

$$
\left.v\right|_{\partial \mathcal{D}} \geq\left. u\right|_{\partial \mathcal{D}}
$$

Indeed, $v \geq 0=u$ on $S(u)$ from the definition of $\mathcal{D}$ and $v$. On $\left\{x_{n}=N_{2}-\delta\right\}$, we apply (5.2) to $u$ and use the fact that $\left.v\right|_{\left\{x_{n}=N_{2}-\delta\right\}}=\left.u\right|_{\left\{x_{n}=N_{2}\right\}}$ to have

$$
v \geq u+\left(q^{-}-q^{+}-M \sigma\right) \delta \geq u+\frac{q^{-}-q^{+}}{2} \delta \quad \text { on }\left\{x_{n}=N_{2}-\delta\right\},
$$

if $\sigma$ is sufficiently small.

Since $\hat{u}$ satisfies (5.4) in $\Omega^{+}(\hat{u})$, then $v$ satisfies

$$
\operatorname{div} A\left(x+\delta \nu_{0}, D v\right)=0 \quad \text { in } \mathcal{D} .
$$


We write this equation in the form:

$$
\operatorname{div} A(x, D v)=\operatorname{div} \psi(x) \quad \text { in } \mathcal{D},
$$

where $A(y, P)$ is the function (5.7) and

$$
\psi(x):=A(x, D v(x))-A\left(x+\delta \nu_{0}, D v(x)\right)=-\delta \int_{0}^{1} D_{y} A\left(x+\delta t \nu_{0}, D v(x)\right) \cdot \nu_{0} d t .
$$

By (5.7) and (2.13), for any $L_{0}>0$,

$$
\sup _{|P| \leq L_{0}}\left\|D_{y} A(\cdot, P)\right\|_{0, \alpha, \Omega_{e}} \leq C\left(L_{0}\right) \sigma, \quad \sup _{|P| \leq L_{0}}\left\|D_{y P}^{2} A(\cdot, P)\right\|_{0,0, \Omega_{e}} \leq C\left(L_{0}\right) \sigma .
$$

From this, we use $|D v| \leq q^{-}-q^{+}+C M \sigma$ with $M \sigma \leq 1$ to conclude

$$
|\psi| \leq C \delta \sigma \quad \text { in } \Omega_{e},
$$

and

$$
\begin{aligned}
|\psi(x)-\psi(\hat{x})| \leq & \delta\left|\int_{0}^{1}\left(D_{y} A\left(x+\delta s \nu_{0}, D v(x)\right)-D_{y} A\left(\hat{x}+\delta s \nu_{0}, D v(x)\right)\right) \cdot \nu_{0} d s\right| \\
& +\delta\left|\int_{0}^{1}\left(D_{y} A\left(\hat{x}+\delta s \nu_{0}, D v(x)\right)-D_{y} A\left(\hat{x}+\delta s \nu_{0}, D v(\hat{x})\right)\right) \cdot \nu_{0} d s\right| \\
\leq & C \delta \sigma|x-\hat{x}|^{\alpha}+C \delta|D v(x)-D v(\hat{x})| \\
\leq & C M \delta \sigma|x-\hat{x}|^{\alpha},
\end{aligned}
$$

where we used (5.2) and $v(x)=\hat{u}\left(x+\delta \nu_{0}\right)$ in the last inequality. Thus, we have

$$
\|\psi\|_{0, \alpha, \Omega_{e}} \leq C M \delta \sigma \text {. }
$$

Then, denoting $w:=v-u$,

$$
\operatorname{div}(A(x, D v)-A(x, D u))=\operatorname{div} \psi(x) \quad \text { in } \mathcal{D}
$$

can be rewritten as

$$
\sum_{i, j=1}^{n} D_{i}\left(a_{i j}(x) D_{j} w\right)=\operatorname{div} \psi(x) \quad \text { in } \mathcal{D}
$$

where $a_{i j}(x)=\int_{0}^{1} A_{P_{j}}^{i}(x, s D v(x)+(1-s) D u(x)) d s$. Thus, equation (5.20) is uniformly elliptic with the ellipticity constants and norms of $a_{i j} \in C^{1, \alpha}(\overline{\mathcal{D}})$ depending only on $n, q^{+}, \gamma$, and $\Omega$.

Step 5. We write $w:=w_{1}+w_{2}$, where $w_{1}$ and $w_{2}$ are the solutions of

$$
\begin{aligned}
\sum_{i, j=1}^{n} D_{i}\left(a_{i j}(x) D_{j} w_{1}\right) & =0 & & \text { in } \mathcal{D}, \\
w_{1} & =w & & \text { on } \partial \mathcal{D},
\end{aligned}
$$

and

$$
\begin{aligned}
\sum_{i, j=1}^{n} D_{i}\left(a_{i j}(x) D_{j} w_{2}\right) & =\operatorname{div} \psi(x) \quad \text { in } \mathcal{D}, \\
w_{2} & =0 \quad \text { on } \partial \mathcal{D} .
\end{aligned}
$$


Indeed, we obtain the solution $w_{1} \in C^{2, \alpha}(\overline{\mathcal{D}})$ of (15.21) in the periodic case $\mathcal{D} \subset$ $\mathbf{T}^{n-1} \times\left[-N_{1}, N_{2}\right]$ by the argument similar to the proof of Step 2 in $\$ 4.2$. Note that $\partial \Omega_{e} \in C^{\infty}, w=v-u \in C^{2, \alpha}$, and the maximum principle:

$$
\sup _{\mathcal{D}} w_{1}=\sup _{\partial \mathcal{D}} w_{1}
$$

which follows from the ellipticity and structure of equation (5.21). As long as $w_{1}$ is obtained, $w_{2}=w-w_{1}$ is a solution of (5.22).

By Theorems $8.16,8.32$, and 8.33 in 17 , which can be adapted to the periodic case $\mathcal{D} \subset \mathbf{T}^{n-1} \times\left[-N_{1}, N_{2}\right]$ without change in the proofs, we have

$$
\left\|w_{2}\right\|_{1, \alpha, \mathcal{D}} \leq C\|\psi\|_{0, \alpha, \Omega_{e}}
$$

where $C$ depends only on the ellipticity constants and $\mathcal{D}$. Furthermore, using (5.15) and $\|f\|_{2, \alpha, \mathbf{T}^{n-1}} \leq C M \sigma$ and choosing $\sigma \leq \min \left(N_{2} / 100,1\right)$ yield that the dependence of $C$ on $\mathcal{D}$ becomes the dependence only on $N_{2}$. Thus, we use (5.19) to obtain

$$
\left\|w_{2}\right\|_{1, \alpha, \mathcal{D}} \leq C M \delta \sigma .
$$

Step 6. Now we estimate $\left(w_{1}\right)_{\nu}\left(x_{*}\right)$ from below. By (5.16), $w_{1} \geq 0$ on $\partial \mathcal{D}$. Thus, $w_{1} \geq 0$ in $\mathcal{D}$ by the maximum principle. Also, $w_{1}\left(x_{*}\right)=0$. Moreover, by (5.17),

$$
w_{1} \geq \frac{q^{-}-q^{+}}{2} \delta \quad \text { on }\left\{x_{n}=N_{2}-\delta\right\} .
$$

We first show that

$$
w_{1}\left(0, \frac{N_{2}}{2}\right) \geq c\left(q^{-}-q^{+}\right) \delta
$$

where $c>0$ depends only on the ellipticity constants $\lambda$ and $\Lambda$ of equation (5.21), i.e., on $q^{+}, q^{-}, \gamma, n$, and $\Omega$.

Since $a_{i j} \in C^{1, \alpha}(\overline{\mathcal{D}})$, equation (5.21) can be rewritten in the nondivergence form:

$$
\sum_{i, j=1}^{n} a_{i j} D_{i j} w_{1}+\sum_{i=1}^{n} b_{i} D_{i} w_{1}=0 \quad \text { in } \mathcal{D}
$$

where $b_{i}:=\sum_{j=1}^{n} a_{i j, x_{i}} \in C^{\alpha}(\overline{\mathcal{D}})$, and $B:=\left(\sum_{i=1}^{n}\left\|b_{i}\right\|_{L^{\infty}(\mathcal{D})}^{2}\right)^{1 / 2}$ depends only on $q^{+}, \gamma, n, \Omega$, and $M$. In the rest of the proof, we consider $\mathcal{D}$ as a subset of $\mathbf{R}^{n-1} \times\left[-N_{1}, N_{2}\right]$ and the functions $w_{1}, a_{i j}$, and $b_{i}$ in (5.25) as the functions on $\mathcal{D}$, $2 a$-periodic with respect to $x_{1}, \cdots, x_{n-1}$. Define a domain $\mathcal{D}_{0}:=(-3 a, 3 a)^{n-1} \times$ $\left(\frac{N_{2}}{4}, N_{2}-\delta\right) \subset \mathcal{D}$, and let $W \in C^{2, \alpha}\left(\mathcal{D}_{0}\right) \cap C\left(\overline{\mathcal{D}_{0}}\right)$ be a solution of

$$
\begin{aligned}
& \mathcal{P}_{\Lambda, \lambda}^{-}\left(D^{2} W\right)-B|D W|=0 \quad \text { in } \mathcal{D}_{0}, \\
& \left.W\right|_{\partial \mathcal{D}_{0}}=\phi,
\end{aligned}
$$

where $\mathcal{P}_{\Lambda, \lambda}^{-}$is the extremal Pucci operator (e.g., [7]), and $\phi \in C^{\alpha}\left(\overline{\mathcal{D}}_{0}\right)$ is a function satisfying

$$
\begin{aligned}
& 0 \leq \phi \leq 1 \quad \text { on } \partial \mathcal{D}_{0}, \\
& \phi=0 \quad \text { on } \partial \mathcal{D}_{0} \backslash\left\{x_{n}=N_{2}-\delta\right\}, \\
& \phi=1 \quad \text { on }\left\{x_{n}=N_{2}-\delta,\left|x^{\prime}\right| \leq a\right\}
\end{aligned}
$$


The existence and regularity of $W$ follow from Chapter 9 in [7], with standard modifications, to take into account the dependence on $D W$ in the equation. Also, $W>0$ in $\mathcal{D}_{0}$, by the strong maximum principle.

Now $w_{1}$, as a solution of (5.25), satisfies

$$
\mathcal{P}_{\Lambda, \lambda}^{-}\left(D^{2} w_{1}\right)-B\left|D w_{1}\right| \leq 0 \quad \text { in } \mathcal{D}
$$

i.e., $w_{1}$ is a supersolution. Also, $w_{1} \geq \frac{q^{-}-q^{+}}{2} W \delta$ on $\partial \mathcal{D}_{0}$. Thus, by the homogeneity of equation (5.26) and the maximum principle, $w_{1} \geq \frac{q^{-}-q^{+}}{2} W \delta$ in $\mathcal{D}_{0}$. Thus, (5.24) is proved with $c=W\left(\frac{N_{2}}{2}, 0\right) / 2>0$ depending only on $\lambda, \Lambda, n$, and $\Omega$.

Step 7. By (5.24) and the interior Harnack inequality applied in $\overline{\mathcal{D}_{0}} \subset \mathcal{D}$, there exists $c>0$ such that

$$
w_{1} \geq c \delta \quad \text { in } \mathcal{D}_{0} \cap\left\{N_{2} / 4 \leq x_{n} \leq 3 N_{2} / 4\right\} .
$$

By the periodicity of $w_{1}$ with respect to $x_{1}, \cdots, x_{n-1}$, we conclude that the above inequality holds in $\mathcal{D} \cap\left\{N_{2} / 4 \leq x_{n} \leq 3 N_{2} / 4\right\}$.

Since $S(u)=\left\{x_{n}=f\left(x^{\prime}\right)\right\}$ and $\|f\|_{2, \alpha, \mathbf{T}^{n-1}} \leq C M \sigma$, it follows that, if $M \sigma$ is small, every point of $S(u)$ has a tangent ball with radius $R \geq N_{2} / 4$ and center within $\left\{N_{2} / 4 \leq x_{n} \leq 3 N_{2} / 4\right\}$. Let $x_{0}$ be the center of such a ball, tangent to $S(u)$ at $x_{*}$, i.e., $B_{R}\left(x_{0}\right) \subset \mathcal{D}$ and $x_{*} \in \partial B_{R}\left(x_{0}\right) \cap \partial \mathcal{D}$. Then, $x_{0} \in\left\{N_{2} / 4 \leq x_{n} \leq 3 N_{2} / 4\right\}$, and thus

$$
w_{1}\left(x_{0}\right) \geq c \delta .
$$

Now the Harnack inequality, applied to $w_{1}$ in $B_{R}\left(x_{0}\right)$, implies

$$
\inf _{B_{R / 2}\left(x_{0}\right)} w_{1} \geq c \delta
$$

By the proof of the Hopf Lemma in [17, Lemma 3.4],

$$
\left(w_{1}\right)_{\nu}\left(x_{*}\right) \geq c_{1} \inf _{B_{R / 2}\left(x_{0}\right)} w_{1},
$$

where $c_{1}>0$ depends only on $R>0, \Lambda, \lambda$, and the $L^{\infty}$-norm of the coefficients of (5.25).

Thus,

$$
\left(w_{1}\right)_{\nu}\left(x_{*}\right) \geq c \delta
$$

where $c>0$ depends only on $n, \gamma, q^{+}, \Omega$, and $M$.

Combining (5.27) with (5.23), we obtain

$$
(v-u)_{\nu}\left(x_{*}\right)=\partial_{\nu}\left(w_{1}+w_{2}\right)\left(x_{*}\right) \geq(c-C M \sigma) \delta \geq \frac{c}{2} \delta,
$$

if $M \sigma$ is small. If $\delta>0$ and $\sigma$ is small, this contradicts (5.14). Thus $\delta=0$, which implies Theorem [5.1.

\section{Stability of Free Boundaries}

As a consequence of the uniqueness, nondegeneracy, and regularity of solutions of the free boundary problem, we have the following stability theorem. 
Theorem 6.1. Let $M>0$. There exist a nonnegative function $\Psi \in C\left(\overline{\mathbf{R}^{+}}\right)$, satisfying $\Psi(0)=0$, and $\sigma_{0}>0$, depending only on $M, q^{+}, q^{-}, \gamma$, and $\Omega$, such that, if $\sigma<\sigma_{0}, \varphi^{-}$satisfies (2.13), and $\hat{\varphi}^{-}$satisfies

$$
\left\|\varphi^{-}-\hat{\varphi}^{-}\right\|_{2, \alpha, \Omega_{e}} \leq \kappa
$$

with $\kappa<\sigma$, then the unique solutions $\varphi$ and $\hat{\varphi}$ of Problem $B$ with (5.1) for $\varphi^{-}$and $\hat{\varphi}^{-}$, respectively, satisfy

$$
\left\|f_{\varphi}-f_{\hat{\varphi}}\right\|_{2, \alpha, \mathbf{T}^{n-1}} \leq \Psi(\kappa),
$$

where $f_{\varphi}$ and $f_{\hat{\varphi}}$ are the "free boundary" functions in (4.4) with $\varphi$ and $\hat{\varphi}$, respectively.

Proof. Let $\sigma_{0}$ be such that Theorem 5.1 holds for $2 \sigma_{0}$. If the assertion is false, then there exist $\varphi_{k}^{-}$and $\hat{\varphi}_{k}^{-}$for $k=1, \cdots$, such that

$$
\begin{aligned}
& \varphi_{k}^{-} \text {satisfy (2.13) with } \sigma \leq \sigma_{0} \\
& \left\|\varphi_{k}^{-}-\hat{\varphi}_{k}^{-}\right\|_{2, \alpha, \Omega_{e}} \leq \frac{1}{k} \\
& \left\|f_{k}-\hat{f}_{k}\right\|_{2, \alpha, \mathbf{T}^{n-1}} \geq \epsilon>0
\end{aligned}
$$

Here $f_{k}$ and $\hat{f}_{k}$ are the "free boundary" functions in (4.4) for $\varphi_{k}$ and $\hat{\varphi}_{k}$, respectively, where $\varphi_{k}$ and $\hat{\varphi}_{k}$ are the unique solutions of Problem $B$ for $\varphi_{k}^{-}$and $\hat{\varphi}_{k}^{-}$, respectively, satisfying (5.1).

By selecting a subsequence (for which we do not change notation), we have

$$
\begin{array}{lr}
\varphi_{k}^{-} \rightarrow \varphi^{-} & \text {in } C^{2, \frac{\alpha}{2}}\left(\overline{\Omega_{e}}\right), \\
\hat{\varphi}_{k}^{-} \rightarrow \varphi^{-} & \text {in } C^{2, \frac{\alpha}{2}}\left(\overline{\Omega_{e}}\right), \\
f_{k} \rightarrow f \quad \text { in } C^{2, \frac{\alpha}{2}}\left(\mathbf{T}^{n-1}\right), & \\
\hat{f}_{k} \rightarrow \hat{f} \quad \text { in } C^{2, \frac{\alpha}{2}}\left(\mathbf{T}^{n-1}\right), \\
f \neq \hat{f},
\end{array}
$$

and $\varphi^{-} \in C^{2, \alpha}\left(\overline{\Omega_{e}}\right)$ satisfies (2.13) with $\sigma \leq \sigma_{0}$ and $f, \hat{f} \in C^{2, \alpha}\left(\mathbf{T}^{n-1}\right)$.

Also, the argument similar to the proof of Proposition 4.5(ii) yields that

$$
\begin{aligned}
& \mathcal{P}_{\varphi_{k}}\left(\left.\varphi_{k}\right|_{\Omega^{+}\left(\varphi_{k}\right)}\right) \rightarrow \mathcal{P}_{\varphi}\left(\left.\varphi\right|_{\Omega^{+}(\varphi)}\right) \quad \text { in } C^{2, \frac{\alpha}{2}}\left(\overline{\Omega_{e}}\right), \\
& \mathcal{P}_{\hat{\varphi}_{k}}\left(\left.\hat{\varphi}_{k}\right|_{\Omega^{+}\left(\hat{\varphi}_{k}\right)}\right) \rightarrow \mathcal{P}_{\hat{\varphi}}\left(\left.\hat{\varphi}\right|_{\Omega^{+}(\hat{\varphi})}\right) \quad \text { in } C^{2, \frac{\alpha}{2}}\left(\overline{\Omega_{e}}\right) \text {. }
\end{aligned}
$$

Here $\varphi \in C\left(\Omega_{e}\right) \cap C^{2, \alpha}\left(\overline{\Omega^{+}(\varphi)}\right)$ is a solution of Problem $B$ for the limiting function $\varphi^{-}$in (6.3) and (6.4), and the "free boundary" function of $\varphi$ is the limiting function $f$ in (6.5); it also follows that $\varphi$ satisfies (5.1). Similarly, $\hat{\varphi} \in C\left(\Omega_{e}\right) \cap C^{2, \alpha}\left(\overline{\Omega^{+}(\varphi)}\right)$ is a solution of Problem $B$ for the limiting function $\varphi^{-}$in (6.3) and (6.4), and the "free boundary" function of $\hat{\varphi}$ is the limiting function $\hat{f}$ in (6.6); it also follows that $\hat{\varphi}$ satisfies (5.1). By (6.7), this contradicts the uniqueness result of Theorem [5.1 for $\varphi^{-}$.

\section{Multidimensional transonic SHOCKS NEAR SPHERICAL AND CIRCULAR SHOCKS}

In this section we are concerned with applications of our approach to the construction of multidimensional transonic shocks with more complex geometries. As 
an example, we focus on multidimensional transonic shocks near spherical $(n \geq 3)$ or circular $(n=2)$ transonic shocks.

We first show the existence of spherical and circular transonic shocks. That is, choosing any

$$
0<R_{1}<R_{0}<R_{2}<\infty,
$$

we consider the domain $\Omega=\left\{x \in \mathbf{R}^{n}: R_{1}<|x|<R_{2}\right\}$ and show that there exists a weak solution $\varphi_{0} \in W^{1, \infty}(\Omega)$ of (1.1) and (1.2) in the sense of (2.1) with $\varphi_{0}(x)=w(|x|)$ for some $w: \mathbf{R} \rightarrow \mathbf{R}$ such that $\varphi_{0} \in C^{\infty}\left(\overline{\Omega_{0}^{ \pm}}\right)$and

$$
\Omega_{0}^{-}=\left\{x \in \mathbf{R}^{n}: R_{1}<|x|<R_{0}\right\} \quad \text { and } \quad \Omega_{0}^{+}=\left\{x \in \mathbf{R}^{n}: R_{0}<|x|<R_{2}\right\}
$$

are respectively supersonic and subsonic regions of $\varphi_{0}$, i.e.,

$$
\begin{aligned}
& \left|D \varphi_{0}\right|>p_{\text {sonic }}^{1} \quad \text { in } \Omega_{0}^{-}, \\
& \left|D \varphi_{0}\right|<p_{\text {sonic }}^{1} \quad \text { in } \Omega_{0}^{+},
\end{aligned}
$$

and $\left|D \varphi_{0}\right|$ has a jump across $S_{0}:=\left\{|x|=R_{0}\right\}$.

It is easy to see that a function $\varphi_{0}(x)=w(|x|)$ satisfies (2.1) in $\Omega$ if and only if, for any $\zeta \in C_{0}^{\infty}\left(R_{1}, R_{2}\right)$,

$$
\int_{R_{1}}^{R_{2}} \rho\left(\left|w^{\prime}(r)\right|^{2}\right) w^{\prime}(r) \zeta^{\prime}(r) r^{n-1} d r=0 .
$$

It follows that $w(|x|)$ is a smooth solution of (1.1) if

$$
\left(\rho\left(\left|w^{\prime}(r)\right|^{2}\right) w^{\prime}(r) r^{n-1}\right)^{\prime}=0 .
$$

Thus,

$$
\rho\left(\left|w^{\prime}(r)\right|^{2}\right) w^{\prime}(r)=\frac{c}{r^{n-1}}
$$

which can be written as

$$
\Phi_{1}\left(w^{\prime}(r)\right)=\frac{c}{r^{n-1}}
$$

where $\Phi_{1}(\cdot)$ is defined in Lemma 2.1, From Lemma 2.1, it follows that there exist smooth functions

$$
\begin{aligned}
& \Phi_{+}^{-1}:\left(0, \Phi_{1}\left(p_{\text {sonic }}^{1}\right)\right) \rightarrow\left(0, p_{\text {sonic }}^{1}\right) \\
& \Phi_{-}^{-1}:\left(0, \Phi_{1}\left(p_{\text {sonic }}^{1}\right)\right) \rightarrow\left(p_{\text {sonic }}^{1}, \sqrt{1 / \theta}\right)
\end{aligned}
$$

which are the inverse functions of $\Phi_{1}(\cdot)$ in the sense that $\Phi_{1}\left(\Phi_{ \pm}^{-1}(\tau)\right)=\tau$ for any $\tau \in$ $\left(0, \Phi_{1}\left(p_{\text {sonic }}^{1}\right)\right)$, such that $\left(\Phi_{+}^{-1}\right)^{\prime}(\tau)>0$ and $\left(\Phi_{-}^{-1}\right)^{\prime}(\tau)<0$ for $\tau \in\left(0, \Phi_{1}\left(p_{\text {sonic }}^{1}\right)\right)$. Thus, in order to satisfy (7.1) and (7.2), we have to choose $c>0$ such that

$$
0<\frac{c}{R_{2}^{n-1}}<\frac{c}{R_{0}^{n-1}}<\frac{c}{R_{1}^{n-1}}<\Phi_{1}\left(p_{\text {sonic }}^{1}\right)
$$

and set

$$
\begin{array}{ll}
w^{\prime}(r)=\Phi_{-}^{-1}\left(\frac{c}{r^{n-1}}\right) & \text { for } R_{1}<r<R_{0}, \\
w^{\prime}(r)=\Phi_{+}^{-1}\left(\frac{c}{r^{n-1}}\right) & \text { for } R_{0}<r<R_{2} .
\end{array}
$$


Thus, we obtain a weak solution $\varphi_{0}(x)=w(|x|)$ of (1.1) in $\Omega$, satisfying (7.1), by setting

$$
\begin{aligned}
& w(r)=-\int_{r}^{R_{0}} \Phi_{-}^{-1}\left(\frac{c}{\tau^{n-1}}\right) d \tau<0 \quad \text { for } R_{1}<r<R_{0}, \\
& w(r)=\int_{R_{0}}^{r} \Phi_{+}^{-1}\left(\frac{c}{\tau^{n-1}}\right) d \tau>0 \quad \text { for } R_{0}<r<R_{2} .
\end{aligned}
$$

On the other hand, we can switch subsonic and supersonic regions of the solution with a spherical or circular shock. Precisely, we can construct another weak solution $\tilde{\varphi}_{0}(x)=\tilde{w}(|x|)$ of (1.1) in $\Omega$ such that

$$
\begin{array}{ll}
\left|D \tilde{\varphi}_{0}\right|>p_{\text {sonic }}^{1} & \text { in } \tilde{\Omega}_{0}^{-}:=\left\{x \in \mathbf{R}^{n}: R_{0}<|x|<R_{2}\right\}, \\
\left|D \tilde{\varphi}_{0}\right|<p_{\text {sonic }}^{1} & \text { in } \tilde{\Omega}_{0}^{+}:=\left\{x \in \mathbf{R}^{n}: R_{1}<|x|<R_{0}\right\} .
\end{array}
$$

Indeed, extend $\Phi_{1}$ to $(-\infty, 0]$ by $\Phi_{1}(-p)=-\Phi_{1}(p)$ for $p>0$. Then we have the inverse functions

$$
\begin{aligned}
& \tilde{\Phi}_{+}^{-1}:\left(-\Phi_{1}\left(p_{\text {sonic }}^{1}\right), 0\right) \rightarrow\left(-p_{\text {sonic }}^{1}, 0\right), \\
& \tilde{\Phi}_{-}^{-1}:\left(-\Phi_{1}\left(p_{\text {sonic }}^{1}\right), 0\right) \rightarrow\left(-\sqrt{1 / \theta},-p_{\text {sonic }}^{1}\right) ;
\end{aligned}
$$

and, following the above argument, we can obtain a solution satisfying (7.3) by defining

$$
\begin{aligned}
& \tilde{w}(r)=\int_{R_{0}}^{r} \tilde{\Phi}_{-}^{-1}\left(-\frac{c}{\tau^{n-1}}\right) d \tau<0 \quad \text { for } R_{0}<r<R_{2}, \\
& \tilde{w}(r)=-\int_{r}^{R_{0}} \tilde{\Phi}_{+}^{-1}\left(-\frac{c}{\tau^{n-1}}\right) d \tau>0 \quad \text { for } R_{1}<r<R_{0},
\end{aligned}
$$

where $c>0$ is as above.

We can express the function $\varphi_{0}$ as

$$
\varphi_{0}(x)=\min \left(\varphi_{0}^{+}(x), \varphi_{0}^{-}(x)\right) \text { for } x \in \bar{\Omega},
$$

where $\varphi_{0}^{ \pm} \in C^{\infty}(\bar{\Omega})$ are defined as

$$
\varphi_{0}^{ \pm}(x)=w_{ \pm}(|x|), \quad w_{ \pm}(r)=\int_{R_{0}}^{r} \Phi_{ \pm}^{-1}\left(\frac{c}{\tau^{n-1}}\right) d \tau, \quad \text { for } R_{1}<r<R_{2} .
$$

The solution $\varphi_{0}$ containing the spherical or circular transonic shock satisfies the entropy condition:

$$
\rho\left(\left|D \varphi_{0}^{-}\right|^{2}\right)<\rho\left(\left|D \varphi_{0}^{+}\right|^{2}\right)
$$

across the transonic shock from the hyperbolic phase to the elliptic phase, which is the direction of fluid motions.

The function $\tilde{\varphi}_{0}$ has the same properties as $\varphi_{0}$.

We now state our results on the existence and stability of multidimensional transonic shocks that are close to the solution $\varphi_{0}$; similar results for $\tilde{\varphi}_{0}$ hold.

Problem $\mathbf{A}^{\prime}$. Given a supersonic solution $\varphi^{-}$of (1.1) in $\Omega$, which is a $C^{2, \alpha}$ perturbation of $\varphi_{0}^{-}$, for some $\alpha>0$ :

$$
\left\|\varphi^{-}-\varphi_{0}^{-}\right\|_{C^{2, \alpha}(\Omega)} \leq \sigma
$$


with $\sigma>0$ small, find a transonic shock solution $\varphi$ in $\Omega$ such that $\varphi=\varphi^{-}$in $\Omega^{-}$, where $\Omega^{-}$is the supersonic region of $\varphi$, and

$$
\begin{array}{lll}
\varphi=\varphi^{-} & \text {on } & \left\{|x|=R_{1}\right\} \\
\varphi=\varphi_{0}^{+} & \text {on } & \left\{|x|=R_{2}\right\} .
\end{array}
$$

Theorem 7.1. There exist positive constants $\sigma_{0}, C_{1}$, and $C_{2}$, depending only on $n, \gamma, c$, and $\Omega$, such that, for every $\sigma \leq \sigma_{0}$ and any supersonic solution $\varphi^{-}$of (1.1) satisfying the conditions stated in Problem $A^{\prime}$, there exists a unique solution $\varphi$ of Problem $A^{\prime}$ satisfying

$$
\left\|\varphi-\varphi_{0}^{+}\right\|_{2, \alpha, \Omega^{+}} \leq C_{1} \sigma,
$$

and $\Omega^{+}=\left\{|x|>f\left(\frac{x}{|x|}\right)\right\} \cap \Omega$, where $f: S^{n-1} \rightarrow \mathbf{R}$, and

$$
\left\|f-R_{0}\right\|_{2, \alpha, S^{n-1}} \leq C_{2} \sigma .
$$

The proof of Theorem 7.1 closely follows the proof of Theorem 2.1. We will only point out some details.

First, we reformulate Problem $\mathrm{A}^{\prime}$ into a free boundary problem. Following the heuristic discussion preceding the statement of Problem B and taking into account the geometry of the present case, we expect that the solution of Problem $\mathrm{A}^{\prime}$ satisfies

$$
\varphi(x) \leq \varphi^{-}(x), \quad \text { for } x \in \Omega \text {. }
$$

Then our free boundary problem is

Problem $\mathbf{B}^{\prime}$. Find $\varphi \in C(\bar{\Omega})$ such that

(i) $\varphi$ satisfies (7.7) in $\Omega$ and (7.6) on $\partial \Omega$;

(ii) $\varphi \in C^{2, \alpha}\left(\overline{\Omega^{+}}\right)$is a solution of (1.1) in $\Omega^{+}=\left\{\varphi(x)<\varphi^{-}(x)\right\} \cap$ $\Omega$, the noncoincidence set;

(iii) the free boundary $S=\partial \Omega^{+} \cap \Omega$ is given by the equation $|x|=$ $f\left(\frac{x}{|x|}\right)$ so that $\Omega^{+}=\left\{|x|>f\left(\frac{x}{|x|}\right)\right\} \cap \Omega$, where $f: S^{n-1} \rightarrow \mathbf{R}$ satisfies $f \in C^{2, \alpha}\left(S^{n-1}\right)$;

(iv) the free boundary conditions (2.3) and (2.4) hold on $S$.

We now solve Problem B', similarly to Problem B above. Namely, we consider the problem with the truncated equation (3.6) and the free boundary condition (3.7). We do not need to extend our domain $\Omega$ here, since we expect that the free boundary should lie in the interior of $\Omega$.

Similar to (4.6), we define

$$
\nu_{0}(x):=\frac{D \varphi_{0}^{+}(x)}{\left|D \varphi_{0}^{+}(x)\right|}=\frac{x}{|x|} .
$$

Now we can follow the argument of Section 4. We clarify the following three points.

First, the nondegeneracy estimate (4.3) is now

$$
\left(\varphi^{-}-\psi\right)_{\nu_{0}}(x) \geq \frac{1}{2}\left(\Phi_{-}^{-1}\left(\frac{c}{R_{2}^{n-1}}\right)-\Phi_{+}^{-1}\left(\frac{c}{R_{1}^{n-1}}\right)\right)>0,
$$


if $\sigma$ is sufficiently small which depends only on $n, q^{+}, \gamma$, and $\Omega$. We obtain this estimate since, for any $x \in \Omega$,

$$
\begin{aligned}
\left(\varphi_{0}^{-}\right)_{\nu_{0}}(x) & =w_{-}^{\prime}(|x|) \geq \Phi_{-}^{-1}\left(\frac{c}{R_{2}^{n-1}}\right)>p_{\text {sonic }}^{1} \\
& >\Phi_{+}^{-1}\left(\frac{c}{R_{1}^{n-1}}\right) \geq w_{+}^{\prime}(|x|) \\
& =\left(\varphi_{0}^{+}\right)_{\nu_{0}}(x),
\end{aligned}
$$

where we used that $\Phi_{+}^{-1}(\cdot)$ is increasing and $\Phi_{-}^{-1}(\cdot)$ decreasing, and $R_{1} \leq|x| \leq R_{2}$.

Second, $A(P)$ in the argument of Section 4.2 is now replaced by $A(x, P)$ with the formula:

$A(x, P)=\tilde{\rho}\left(\left|P+D \varphi_{0}^{+}(x)\right|^{2}\right)\left(P+q^{+} D \varphi_{0}^{+}(x)\right)-\rho\left(\left|D \varphi_{0}^{+}(x)\right|^{2}\right) D \varphi_{0}^{+}(x) \quad$ for $P \in \mathbf{R}^{n}$, and $g_{\psi}(x)$ is defined by (4.18), where $\varphi_{0}^{+}(x)$ is now defined by (7.4).

Third, (4.19) still holds in $\Omega$. Indeed, since $D \varphi_{0}^{ \pm}(x)=w_{ \pm}^{\prime}(|x|) \nu_{0}(x)$, we use the function $\Phi_{1}(\cdot)$ from Lemma 2.1 to obtain

$$
\rho\left(\left|D \varphi_{0}^{ \pm}(x)\right|^{2}\right) D \varphi_{0}^{ \pm}(x)=\Phi_{1}\left(\left|w_{ \pm}^{\prime}(|x|)\right|\right) \nu_{0}(x)=\Phi_{1}\left(\Phi_{ \pm}^{-1}\left(\frac{c}{|x|^{n-1}}\right)\right) \frac{x}{|x|}=c \frac{x}{|x|^{n}} .
$$

The uniqueness and stability results, similar to Theorem 6.1, can also be established (see [9]).

\section{Appendix A. Proof of Lemma 4.3}

Consider first the case of the Laplace equation with the Neumann condition on $S_{\psi}$ :

$$
\begin{cases}\Delta u=r & \text { in } \Omega^{+}(\psi) \\ u_{\nu}=g & \text { on } S_{\psi} \\ u=0 & \text { on } \Gamma_{1}\end{cases}
$$

Let $\psi \in C^{\infty}\left(\Omega_{e}\right) \cap \mathcal{K}_{M}$ and $r, g \in C^{\infty}\left(\overline{\Omega^{+}(\psi)}\right)$. We can easily construct $\tilde{g} \in$ $C^{\infty}\left(\overline{\Omega^{+}(\psi)}\right)$ satisfying $\tilde{g}=g$ near $S_{\psi}$ and $\tilde{g}=0$ on $\Gamma_{1}$. Then the function $v:=u-\tilde{g}$ satisfies

$$
\left\{\begin{array}{l}
\Delta v=h:=r-\Delta \tilde{g} \quad \text { in } \Omega^{+}(\psi) \\
v_{\nu}=0 \quad \text { on } S_{\psi}, \\
v=0 \quad \text { on } \Gamma_{1} .
\end{array}\right.
$$

We construct a variational solution of A.2, that is, we minimize a functional

$$
I[w]=\int_{\Omega^{+}(\psi)}\left(|\nabla w|^{2}-h w\right) d x
$$

over the set $\left\{w \in H^{1}\left(\Omega^{+}(\psi)\right):\left.w\right|_{\Gamma_{1}}=0\right\}$, where $\Omega^{+}(\psi) \subset \mathbf{T}^{n-1} \times\left[-N_{1}, N_{2}\right]$ is a compact Riemannian manifold with boundary, and $H^{1}\left(\Omega^{+}(\psi)\right)$ is the Sobolev space, and the condition $\left.w\right|_{\Gamma_{1}}=0$ is understood in the sense of traces. The existence of a solution $v$ follows from the argument similar to the proof of Theorem 4.8 in 4], with the aid of Lemma 4.2 Now a standard regularity theory implies that $v \in C^{\infty}\left(\overline{\Omega^{+}(\psi)}\right)$ and $v$ satisfies $(\overline{\mathrm{A} .2})$ : Indeed, since we can consider $v(x)$ as a periodic solution with respect to the $x^{\prime}$ variables in $\mathbf{R}^{n-1} \times\left[-N_{1}, N_{2}\right] \cap\left\{x_{n}>f\left(x^{\prime}\right)\right\}$, then the interior regularity follows from Theorem 8.10 in [17] and the regularity 
near $\Gamma_{1}$ from Section 8.4 in [17]; and the regularity near $S_{\psi}$ follows from the interior regularity by the local flattening of $S_{\psi}$ and then by the reflection.

Now $u=v+\tilde{g}$ is a $C^{\infty}$ solution of (A.1). Then $u$ satisfies (4.23) with $C$ depending only on the domain $\Omega$.

If $\psi \in \mathcal{K}_{M}, r \in C^{\alpha}\left(\overline{\Omega^{+}(\psi)}\right)$, and $g \in C^{1, \alpha}\left(\overline{\Omega^{+}(\psi)}\right)$, we approximate them by smooth functions $\psi_{j}, r_{j}$, and $g_{i}$, and we use (4.23) to pass to the limit.

Thus we have shown that A.1 is uniquely solvable for $r \in C^{\alpha}\left(\overline{\Omega^{+}(\psi)}\right)$ and $g \in C^{1, \alpha}\left(\overline{\Omega^{+}(\psi)}\right)$, and the solution satisfies 4.23 .

The proof of the existence of a solution for the general problem (4.21) can be achieved via the method of continuity by considering the following problem for $t \in[0,1]:$

$$
\left\{\begin{array}{l}
\overline{\mathcal{L}}_{t}[u]=t \overline{\mathcal{L}} u+(1-t) \Delta u=r \quad \text { in } \Omega^{+}(\psi), \\
t b \cdot D u+(1-t) u_{\nu}=g \quad \text { on } S_{\psi}, \\
v=0 \text { on } \Gamma_{1},
\end{array}\right.
$$

which has the structure of (4.21) with the same ellipticity and obliqueness constants. The argument just repeats the proof of Theorem 6.8 in [17], with the aid of Lemma 4.2 and 4.23).

\section{Appendix B. Proof of Lemma 4.4}

We now prove Lemma 4.4 for the estimate (4.33) in several steps. Denote $g:=$ $t g_{\psi}$.

First, we note that $u(x)$ is a solution of the problem 4.21 with $r(x) \equiv 0$, $a_{i j}(x)=A_{i j}(D u(x))$, and $b_{i}(x)=\sum_{j=1}^{n} \int_{0}^{1} A_{P_{i}}^{j}(s D u(x)) d s \nu^{j}(x)$, where we used that $A(0)=0$. Then $a_{i j}, b_{i} \in C^{1, \alpha}$. Also the uniform ellipticity of $a_{i j}(x)$ is obvious, and the strict obliqueness of $b_{i}(x)$ follows from

$$
b(x) \cdot \nu(x)=\sum_{i, j=1}^{n} \int_{0}^{1} A_{P_{i}}^{j}(s D u(x)) \nu^{i}(x) \nu^{j}(x) d s \geq \lambda>0,
$$

where $\lambda$ and $\Lambda$ are the ellipticity constants of (3.6). Then, by Lemma 4.2(1),

$$
\|u\|_{0, \Omega^{+}(\psi)} \leq C\|g\|_{0, \Omega^{+}(\psi)},
$$

where $C$ depends only on $\lambda, \Lambda$, and $\Omega$. Noting also that $\underline{u}(x)$ and $\bar{u}(x)$ constructed in the proof of Lemma 4.2 are the barriers for $u(x)$ on $\Gamma_{1}$, we have

$$
|D u| \leq C\|g\|_{0, \Omega^{+}(\psi)} \quad \text { on } \Gamma_{1} .
$$

Thus we can combine the estimates for the oblique derivative problem with the estimates for the Dirichlet problem from Chapters 8 and 14 in [17] to prove (4.33).

For the oblique derivative problem, we use the estimates from Sections 2-4 in [24]. Note that 4.29) is the structure condition which allows us to apply these estimates. Also note that the conormal boundary condition on $S_{\psi}$ satisfies the nonlinear strict obliqueness property of [24]: Indeed, denoting $G(x, P):=\sum_{i=1}^{n} A^{i}(P) \nu_{i}(x)$ for $x \in S_{\psi}$ and $P \in \mathbf{R}^{n}$, we obtain $\chi:=G_{P} \cdot \nu=\sum_{i, j=1}^{n} A_{P_{j}}^{i} \nu_{i} \nu_{j} \geq \lambda>0$, and this with the properties (4.17) and (4.29) implies that $G(x, P)$ satisfies the conditions (G2) and (G3) of [24]. Thus, using the regularized distance to $S_{\psi}$ described in [24] page 522], and using Lemmas 3.1 and 3.3 in [24] (with modifications outlined on pages 522-523) and Theorem 4.1 in [24] (with the aid of Lemma 2.2 in [24]), we 
obtain (4.33) in a boundary neighborhood of $S_{\psi}$, where $\delta>0$ is from Theorem 4.1 in [24]. The interior gradient bound follows from Lemma 3.1 in [24].

These estimates combined with (B.1), (B.2), and the estimates of Chapter 13 in [17] yield (4.33) with $C$ depending only on $\|g\|_{1, \alpha, \Omega^{+}(\psi)}, S_{\psi}$, and $\Omega$.

\section{ACKNowledgments}

The authors thank Luis Caffarelli for stimulating conversations. Gui-Qiang Chen's research was supported in part by the National Science Foundation under Grants DMS-0204225, DMS-0204455, and INT-9987378. Mikhail Feldman's research was supported in part by the National Science Foundation under Grants DMS-0096090 and DMS-0074037.

\section{REFERENCES}

1. H. W. Alt and L. A. Caffarelli, Existence and regularity for a minimum problem with free boundary, J. Reine Angew. Math. 325 (1981), 105-144. MR 83a:49011

2. H. W. Alt, L. A. Caffarelli, and A. Friedman, A free boundary problem for quasilinear elliptic equations, Ann. Scuola Norm. Sup. Pisa Cl. Sci. (4), 11 (1984), 1-44. MR 86c:49003

3. H. W. Alt, L. A. Caffarelli, and A. Friedman, Compressible flows of jets and cavities, J. Diff. Eqs. 56 (1985), 82-141. MR 86i:35036

4. T. Aubin, Some Nonlinear Problems in Riemannian Geometry, Springer-Verlag: Heidelberg, 1998. MR 99i:58001

5. L. Bers, Existence and uniqueness of subsonic flows past a given profile, Comm. Pure Appl. Math. 7 (1954), 441-504. MR 16:417a

6. L. A. Caffarelli, A Harnack inequality approach to the regularity of free boundaries, I. Lipschitz free boundaries are $C^{1, \alpha}$, Rev. Mat. Iberoamericana, 3 (1987), 139-162; II. Flat free boundaries are Lipschitz, Comm. Pure Appl. Math. 42 (1989), 55-78; III. Existence theory, compactness, and dependence on X, Ann. Scuola Norm. Sup. Pisa Cl. Sci. (4), 15 (1989), 583-602. MR 90d:35036, MR 90b:35246. MR 91a:35170

7. L. A. Caffarelli and X. Cabre, Fully Nonlinear Elliptic Equations, Colloquium Publications, 43, AMS: Providence, RI, 1995. MR 96h:35046

8. S. Canić, B. L. Keyfitz, and G. Lieberman, A proof of existence of perturbed steady transonic shocks via a free boundary problem, Comm. Pure Appl. Math. 53 (2000), 484-511. MR 2001m:76056

9. G.-Q. Chen and M. Feldman, Steady transonic shocks and free boundary problems in infinite cylinders for the Euler equations, Preprint, Northwestern University, May 2002; Transonic shocks and one-phase free boundary problems for nonlinear equations of mixed elliptichyperbolic type in unbounded domains, Preprint, Northwestern University, November 2002.

10. G.-Q. Chen and J. Glimm, Global solutions to the compressible Euler equations with geometrical structure, Commun. Math. Phys. 180 (1996), 153-193. MR 97j:35120

11. S.-X. Chen, Existence of stationary supersonic flows past a point body, Arch. Rational Mech. Anal. 156 (2001), 141-181. MR 2001m:76058

12. S.-X. Chen, Asymptotic behavior of supersonic flow past a convex combined wedge, Chinese Ann. Math. 19B (1998), 255-264. MR 2000b:76058

13. R. Courant and K. O. Friedrichs, Supersonic Flow and Shock Waves, Springer-Verlag: New York, 1948. MR 10:637c

14. C. M. Dafermos, Hyperbolic Conservation Laws in Continuum Physics, Springer-Verlag: Berlin, 2000. MR 20001m:35212

15. G. Dong, Nonlinear Partial Differential Equations of Second Order, Transl. Math. Monographs, 95, AMS: Providence, RI, 1991. MR 93f:35002

16. R. Finn and D. Gilbarg, Three-dimensional subsonic flows and asymptotic estimates for elliptic partial differential equations, Acta Math. 98 (1957), 265-296. MR 19:1179a

17. D. Gilbarg and N. Trudinger, Elliptic Partial Differential Equations of Second Order, 2nd Ed., Springer-Verlag: Berlin, 1983. MR 86c:35035

18. J. Glimm and A. Majda, Multidimensional Hyperbolic Problems and Computations, SpringerVerlag: New York, 1991. MR 91h:00031 
19. C.-H. Gu, A method for solving the supersonic flow past a curved wedge (in Chinese), Fudan J. 7 (1962), 11-14.

20. C. Kenig, Harmonic Analysis Techniques for Second Order Elliptic Boundary Value Problems, CBMS-RCSM, 83, Amer. Math. Soc.: Providence, RI, 1994. MR 96a:35040

21. P. D. Lax, Hyperbolic Systems of Conservation Laws and the Mathematical Theory of Shock Waves, CBMS-RCSM, SIAM: Philadelphia, 1973. MR 50:2709]

22. T.-T. Li, On a free boundary problem, Chinese Ann. Math. 1 (1980), 351-358. MR 82i:35125

23. G. Lieberman, Regularity of solutions of nonlinear elliptic boundary value problems, J. Reine Angew. Math. 369 (1986), 1-13. MR 87m:35094

24. G. Lieberman and N. Trudinger, Nonlinear oblique boundary value problems for nonlinear elliptic equations, Trans. Amer. Math. Soc. 295 (1986), 509-546. MR 87h:35114

25. W.-C. Lien and T.-P. Liu, Nonlinear stability of a self-similar 3-dimensional gas flow, Commun. Math. Phys. 204 (1999), 525-549. MR 2000f:76016

26. A. Majda, The stability of multidimensional shock fronts, Mem. Amer. Math. Soc. 275; The existence of multidimensional shock fronts, Mem. Amer. Math. Soc. 281, AMS: Providence, 1983. MR 84e:35100. MR 85f:35139

27. C. S. Morawetz, On the nonexistence of continuous transonic flows past profiles I,II,III, Comm. Pure Appl. Math. 9 (1956), 45-68; 10 (1957), 107-131; 11 (1958), 129-144. MR 17:1149d, MR 19:490e, MR 20:2961

28. C. S. Morawetz, Potential theory for regular and Mach reflection of a shock at a wedge, Comm. Pure Appl. Math. 47 (1994), 593-624. MR 95g:76030

29. D. G. Schaeffer, Supersonic flow past a nearly straight wedge, Duke Math. J. 43 (1976), 637-670. MR 54:1850

30. M. Shiffman, On the existence of subsonic flows of a compressible fluid, J. Rational Mech. Anal. 1 (1952), 605-652. MR 14:510b

31. E. M. Stein, Singular Integrals and Differentiability Properties of Functions, Princeton Univ. Press: Princeton, NJ, 1970. MR 44:7280

32. Y. Zhang, Global existence of steady supersonic potential flow past a curved wedge with a piecewise smooth boundary, SIAM J. Math. Anal. 31 (1999), 166-183. MR 2000j:76125

Department of Mathematics, Northwestern University, Evanston, Illinois 60208-2730

E-mail address: gqchen@math.northwestern.edu

Department of Mathematics, University of Wisconsin, Madison, Wisconsin 53706

E-mail address: feldman@math.wisc.edu 